\title{
Due esempi di didattica sulla rappresentazione di relazioni che legano opere, architetti e correnti culturali
}

\author{
Agostino Urso \\ Francesco De Lorenzo
}

\section{Abstract}

Ispirata al Teorema del Modello di Architettura di Roberto Migliari, circoscritta alla rappresentazione codificata dai 12 Gradi di Iconicità di Abraham Moles, la nostra ricerca propone il 'Disegno Complesso' come strumento privilegiato di confronto nelle dinamiche del corso di studio. Utilizzando esemplificativamente l'esperienza del Movimento Moderno, nell'interpretazione di Mies van der Rohe (la sedia Barcelona e il Padiglione tedesco all'Esposizione Universale del 1929) e di Gerrit Thomas Rietveld (la sedia Red\&Blue e la casa Schröder), si racconta una forma di pensiero pervasivo, integrato e totalizzante, sintetizzato dal Bauhaus nel motto "dal cucchiaio alla città": un sistema di progettazione unitario, frutto di una rigorosa analisi funzionale. Concludendo, allora, è demandato al 'Disegno Complesso' il compito di integrare l'analisi dell'artefatto alla descrizione delle idee e dei processi sottesi, procedendo nell'affrontare la lettura del tema indagato in tre livelli di approfondimento. In primo, il più generale, ricerca le relazioni che intercorrono tra il Movimento Moderno e l'intera produzione culturale del XX secolo. II secondo, intermedio, si occupa delle relazioni che intercorrono tra i due architetti, le opere scelte e le rispettive correnti di pensiero. II terzo ed ultimo analizza, seguendo le indicazioni del Bauhaus, quel sistema di progettazione integrato e unitario applicabile sia al design che all'architettura.

Parole chiave

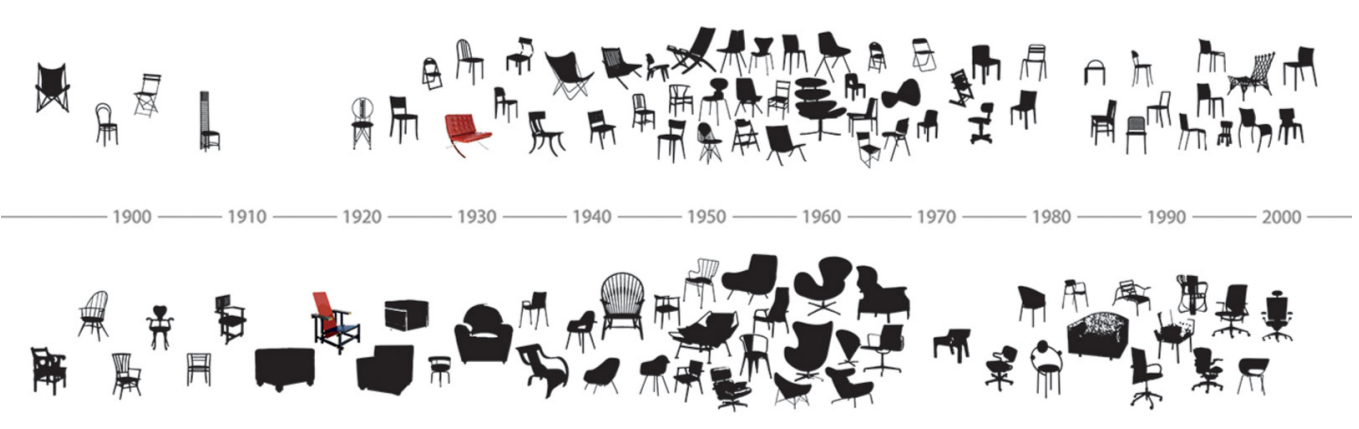




\section{Una constatazione}

È chiaro, a chiunque insegni da un certo numero di anni, quanto siano cambiate le dinamiche di insegnamento nei corsi di studio. Non è possibile affrontare la mutazione verificatasi nell'interazione tra docente e discente senza entrare nel merito dei cambiamenti sociali sperimentati negli ultimi anni. Al mito della velocità si è sostituito quello della simultaneità nel quale è ormai istantanea e globale la condivisione delle informazioni in rete. Riducendo queste dinamiche a due immagini iconiche, abbiamo da un lato il docente, riconducibile al paradigma della radice, dall'altro lo studente, plasticamente definito dall'immagine di rete neurale. II primo incarna la tradizionale attitudine dello studioso ad approfondire ogni tema trattato, per poi sintetizzare e sistematizzare le reciproche relazioni formali e funzionali. II discente procede in maniera opposta: meno propenso alla riflessione, utilizza la rete per produrre veloci manipolazioni di informazioni sul tema indagato, spesso basate su differenti visioni culturali. Concludendo, quindi, se il primo utilizza un approccio incrementale alla conoscenza, il secondo preferisce un metodo di studio basato sulla ricerca di significazioni e connessioni generate dalle reti di informazioni, per poi, all'occorrenza, approfondire.

\begin{tabular}{|c|c|c|c|}
\hline \multicolumn{4}{|c|}{ Escala de Iconicidad decreciente de Abraham Moles } \\
\hline Nivel & Definición & Criterio & Ejemplos \\
\hline 12 & $\begin{array}{l}\text { El referente fisico } \\
\text { mismo }\end{array}$ & Iconicidad. & $\begin{array}{l}\text { Objeto en vitrina o en } \\
\text { exposición }\end{array}$ \\
\hline 11 & $\begin{array}{l}\text { Modelo bi-o } \\
\text { tridimensional a } \\
\text { escala }\end{array}$ & Colores y materiales arbitrarios & $\begin{array}{l}\text { Reconstrucción ficticia, } \\
\text { maqueta }\end{array}$ \\
\hline 10 & $\begin{array}{l}\text { Esquema bi- o } \\
\text { tridimensional } \\
\text { reducido o } \\
\text { aumentado }\end{array}$ & $\begin{array}{l}\text { Colores y materiales escogidos según } \\
\text { criterios lógicos }\end{array}$ & $\begin{array}{l}\text { Mapas en } 3 \\
\text { dimensiones,globo terráqueo }\end{array}$ \\
\hline 9 & $\begin{array}{l}\text { Fotografia o } \\
\text { proyección realista } \\
\text { co un plano }\end{array}$ & $\begin{array}{l}\text { Proyeceión perspectiva rigurosa, medios } \\
\text { tonos y sombras }\end{array}$ & Catálogos ilustrados; posters \\
\hline 8 & $\begin{array}{l}\text { Dibujo, fotografia de } \\
\text { alto contraste }\end{array}$ & $\begin{array}{l}\text { Continuidad del contorno y cierre de la } \\
\text { forma }\end{array}$ & $\begin{array}{l}\text { Afiches, catálogos, } \\
\text { fotografias técnicas }\end{array}$ \\
\hline 7 & Esquema anatómico & $\begin{array}{l}\text { Corte en la carocería o envoltorio; } \\
\text { respeto por la topograffa; cuantificación } \\
\text { de elemientos y simplificación }\end{array}$ & $\begin{array}{l}\text { Corte anatómico, corte de un } \\
\text { motor, plano de conexiones } \\
\text { eléctricas, mapa geográfico }\end{array}$ \\
\hline 6 & $\begin{array}{l}\text { Representación } \\
\text { "estallada" }\end{array}$ & $\begin{array}{l}\text { Disposición perspecpectiva artificial de } \\
\text { piezas según sus relaciones de vecindad } \\
\text { topográfica }\end{array}$ & $\begin{array}{l}\text { Objetos técnicos en } \\
\text { manuales de ensamble o } \\
\text { reparación }\end{array}$ \\
\hline 5 & $\begin{array}{l}\text { Esquema de } \\
\text { principio }\end{array}$ & $\begin{array}{l}\text { Substitución de los componentes por } \\
\text { símbolos normalizados; paso de la } \\
\text { topografia a la topologgaigeometrización }\end{array}$ & $\begin{array}{l}\text { Mapa de conexiones de un } \\
\text { receptor de TV, mapa } \\
\text { esquematizado del Metro }\end{array}$ \\
\hline 4 & $\begin{array}{l}\text { Organigrama o } \\
\text { esquema de bloque }\end{array}$ & $\begin{array}{l}\text { Los clementos son cajas negras" } \\
\text { funcionales, conectadas lögicamente; } \\
\text { presentación de funciones lógicas }\end{array}$ & $\begin{array}{l}\text { Organigrama de una } \\
\text { empresa, flujograma de un } \\
\text { programa computacional }\end{array}$ \\
\hline 3 & $\begin{array}{l}\text { Escuema de } \\
\text { formulación }\end{array}$ & $\begin{array}{l}\text { Relación lógica, no topológica, en un } \\
\text { espacio no geométrico, entre elementos } \\
\text { abstractos. Los lazos son simbólicos y } \\
\text { todos los componentes, visibles. }\end{array}$ & $\begin{array}{l}\text { Fórinulas quimicas } \\
\text { desarrolladas, sociogramas }\end{array}$ \\
\hline 2 & $\begin{array}{l}\text { Esquema en } \\
\text { espacios complejos }\end{array}$ & $\begin{array}{l}\text { Combinación en un mismo espacio de } \\
\text { tepresentación de elementos } \\
\text { esquemáticos pertenecientes a sistemas } \\
\text { diferentes }\end{array}$ & $\begin{array}{l}\text { Fuerzas y posiciones } \\
\text { geométricas en una } \\
\text { estructura metálica; esquema } \\
\text { de estática; representación } \\
\text { sono }\end{array}$ \\
\hline 1 & \begin{tabular}{|l|} 
Esquema de vectores \\
en espacios \\
puramente \\
abstractos
\end{tabular} & $\begin{array}{l}\text { Representación gráfica en un espacio } \\
\text { métrico abstracto, de relaciones entre } \\
\text { tamaños vectoriales }\end{array}$ & $\begin{array}{l}\text { Gráficos vectoriales en } \\
\text { electrotécnica }\end{array}$ \\
\hline 0 & \begin{tabular}{|l|} 
Descripción en \\
palabras \\
normalizadas o \\
fórmulas algebráicas \\
\end{tabular} & $\begin{array}{l}\text { Signos abstractos sin conexión } \\
\text { imaginable con el significado }\end{array}$ & Ecuaciones y textos. \\
\hline
\end{tabular}




\section{Due osservazioni}

Prima osservazione. Nella nostra personale esperienza, capita spesso che, assegnando un esercizio di analisi e ridisegno di un capolavoro di architettura, fornendo agli studenti solo gli estremi bibliografici, gli stessi, in una buona parte dei casi, ritengano evaso il compito assegnato con la restituzione dei canonici disegni bidimensionali e tridimensionali. Solo in pochi casi si propongono ulteriori elaborati di approfondimento in relazione allo studio di simmetrie, ritmi e proporzioni. Quasi mai, infine, si ragiona sul quadro più ampio di relazioni che legano l'artefatto al pensiero del suo creatore o ancora al movimento di idee al quale l'architetto è ascrivibile. L'esercizio di analisi dell'architettura assegnata, quindi, in un significativo numero di casi, coincide con la sua restituzione metrica.

Seconda osservazione. Riferendoci ancora al compito assegnato, quasi mai viene colta la sostanziale differenza che intercorre tra l'analisi e la restituzione di un capolavoro d'architettura e un equivalente manufatto edile realizzato a 'regola d'arte'. È necessario, a quel punto, aprire una riflessione in merito alla definizione di opera d'arte e quindi di manufatto architettonico di rilevante interesse culturale. Diventa quindi utile specificare come l'analisi di un capolavoro, oltre a tenere in conto gli aspetti dimensionali, strutturali, materici e funzionali dell'edificio, debba considerare quei processi culturali, portatori di valori immateriali, il cui profondo appeal è legato all'essere ad un tempo riferimento, memoria e simbolo di un intero sistema sociale o, comunque, di una sua parte politica, istituzionale o culturale.

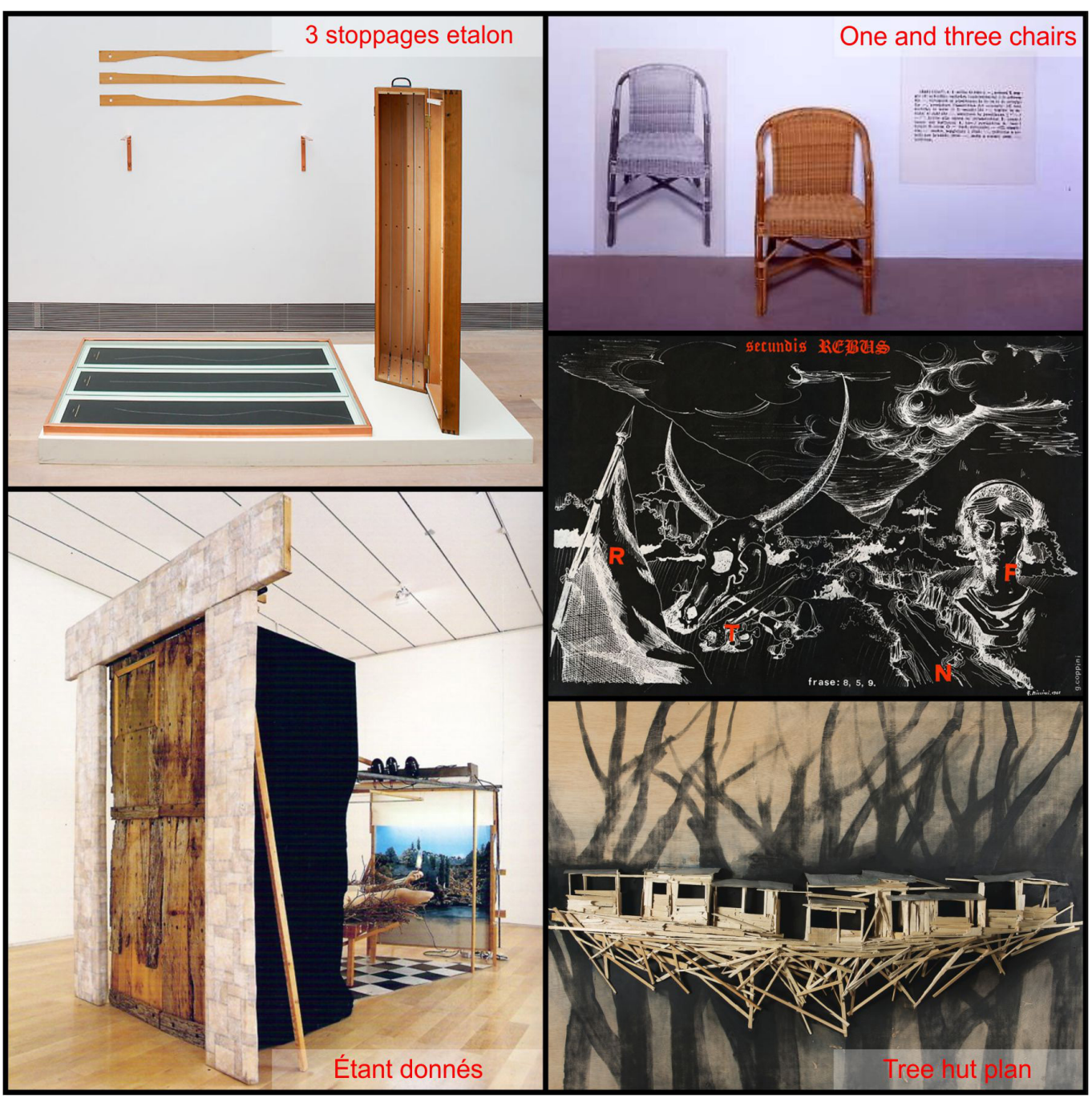




\section{Rappresentazione simultanea degli aspetti materiali e immateriali di un'architettura}

Da un lato i due paradigmi, la radice e la rete neurale, corrispondenti ad altrettanti metodi di acquisizione della conoscenza, dall'altro una pratica del rilievo intesa più che come acquisizione di dati metrici, come restituzione del sistema di relazioni immateriali veicolate. Immaginiamo a questo punto di poter ricondurre l'insieme dei punti di vista esercitati e delle pratiche utilizzate ad una rappresentazione sintetica che consideri tanto gli aspetti fisici, quanto quelli percettivi e concettuali: un approccio multimediale basato sulla lettura contemporanea del maggior numero di elaborazioni grafiche prodotte. Metodologia, semplificando molto, che possiamo definire come somma di due processi: uno teorico, l'altro tecnico. II primo promuove la contaminazione delle conoscenze attraverso un confronto multidisciplinare. II secondo individua, nel Teorema del Modello di Architettura [Migliari 2004, pp. 9, I0] [I], un sistema complesso di rappresentazioni integrate. L'enunciato del teorema, in sostanza, si potrebbe così interpretare: la corrispondenza tra il modello multimediale di sintesi - che da questo punto in poi definiamo 'Disegno Complesso' - ed il suo artefatto crescerà in funzione dell'incremento del numero dei media utilizzati e delle rispettive rappresentazioni sistematizzate.

D'altro canto, la varietà dei media disponibili e la loro sovrapponibilità pone un serio interrogativo sui gradi possibili di rappresentazione della realtà.

Per R. Arnheim rappresentare la realtà è essenzialmente un problema di comprensione visiva della forma, sia essa fisica o percettiva, da ricondurre ai significati che può esprimere ed ai contenuti che intende comunicare.
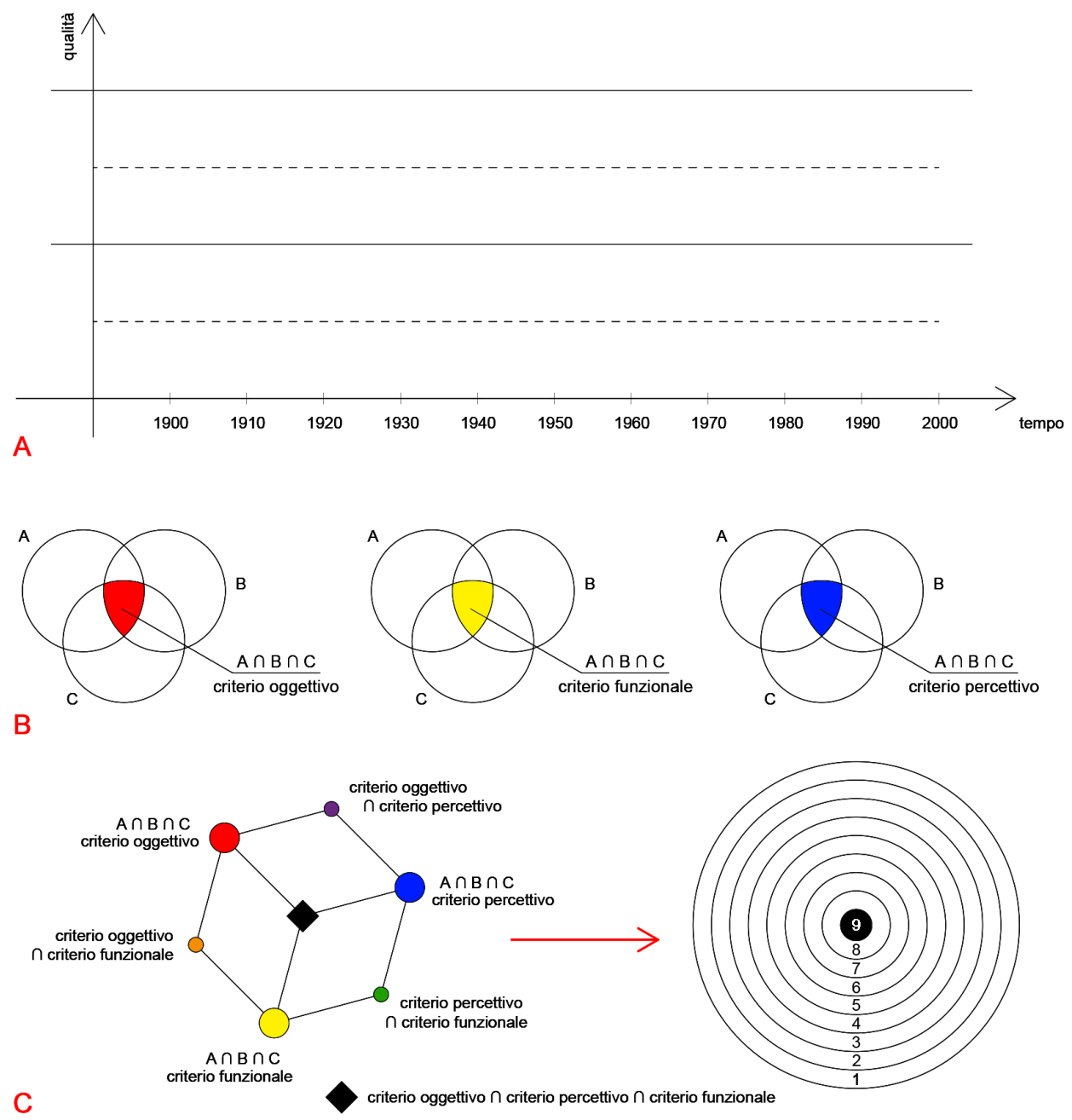
Matt Madden, invece, attraverso il lavoro di Esercizi di stile. 99 modi di raccontare una storia, propone una interessante riflessione su quale rapporto di verità esista tra l'oggetto e le sue rappresentazioni. Una soluzione al tema, già affrontato nel XIX secolo da Peirce (con le definizioni icona, simbolo, indice), viene proposta da Anceschi attraverso la formulazione di una teoria unificante che includa la scala di iconicità proposta da Abraham Moles. Nei suoi 12 gradi sono comprese altrettante variazioni di rappresentazioni ai cui estremi si posizionano l'oggetto reale e la sua controparte astratta, la parola scritta (fig. I). Abbiamo a questo punto una classificazione internazionalmente riconosciuta, pronta ad essere utilizzata per rappresentare gli aspetti tangibili e intangibili di qualsiasi artefatto.

\section{Arte, rappresentazione, multimedialità e interazione}

Elaborata negli anni 1913-1914 da Marcel Duchamp, l'opera 3 stoppages étalon è una riflessione sul passaggio dalla condizione di realtà a quella di virtualità. Passaggio che produce tre interessanti conseguenze: scaricare il soggetto rappresentato da ogni sua valenza iconica; spostarne l'attenzione sulle sue relazioni formali; provocare nell'osservatore una sensazione di straniamento percettivo.

La serie Rebus - iniziata nel 1964 da E. Miccini - allo stesso tempo conclude la riflessione sulla multimedialità e introduce quella su un approccio concettuale all'interattività. La serie Rebus si può definire come una sperimentazione che, attraverso continui rimandi tra immagini e parole, utilizza il collage come strumento di comunicazione della poesia visiva. Se i loro messaggi producono apparentemente un facile ed immediato consenso, solo un coinvolgimento attivo del suo fruitore ne consente la decifrazione del messaggio più vero e profondo.

Nell'anno 1965, J. Kosuth presenta l'installazione One and three chairs. Tre sono gli elementi che la compongono: una sedia, la sua fotografia in posizione frontale e una didascalia con la sua descrizione testuale. Scrive Prestinenza Puglisi: "Scegliendo un oggetto banale e moltiplicandolo per tre, Kosuth lo scarica di ogni valore iconico, con la conseguenza che il suo

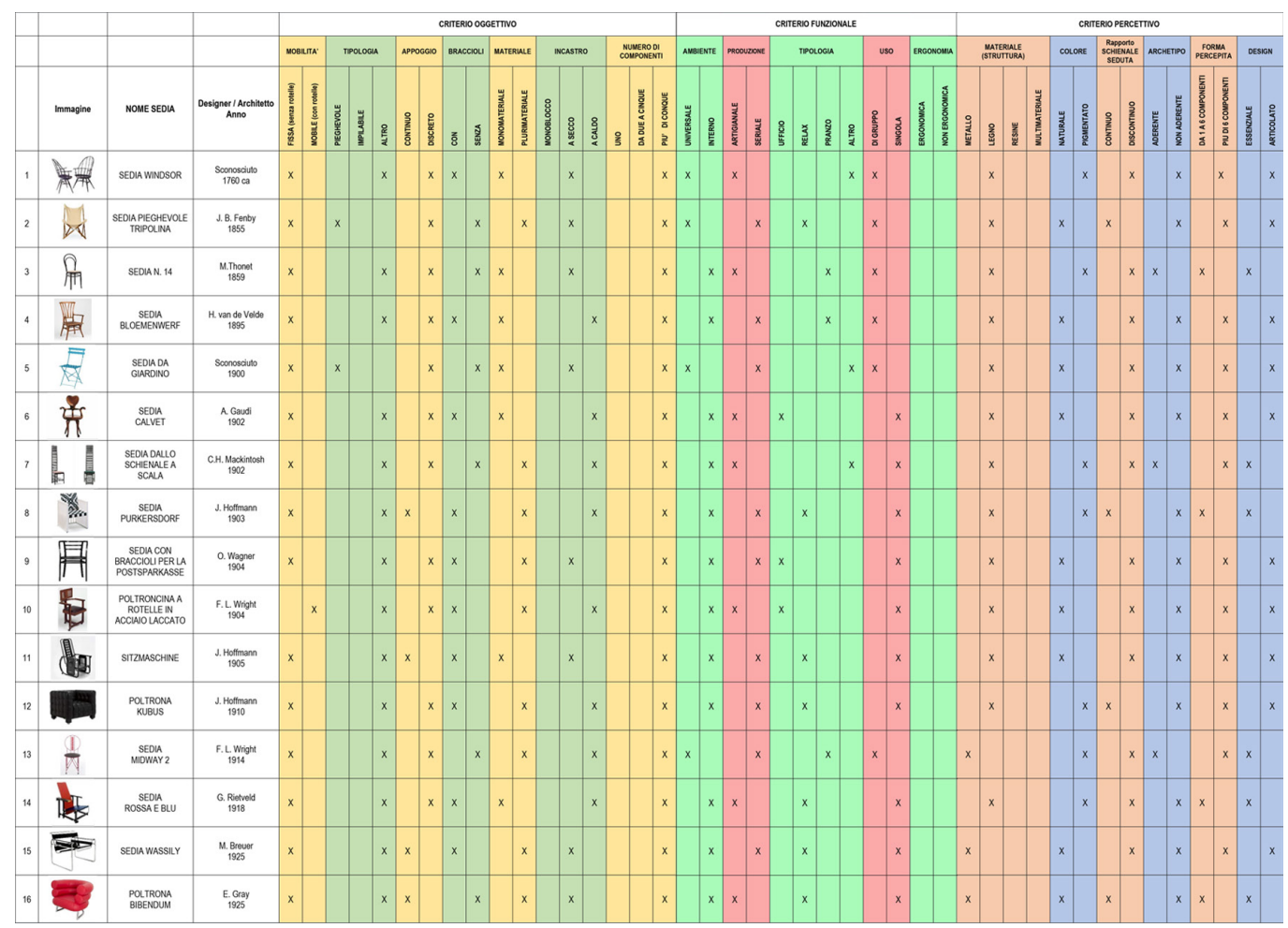


Fig. 5. Grafico cartesiano in XY delle I54 sedie. In ascissa il fattore 'tempo' 'tipologia di materiale' appoggio-braccioli' (B).

Fig. 6. Diagrammi di Eulero-Venn - insieme intersezione. Attraverso gli insiemi si mettono in relazione le 154 sedie con i criteri prescelti.

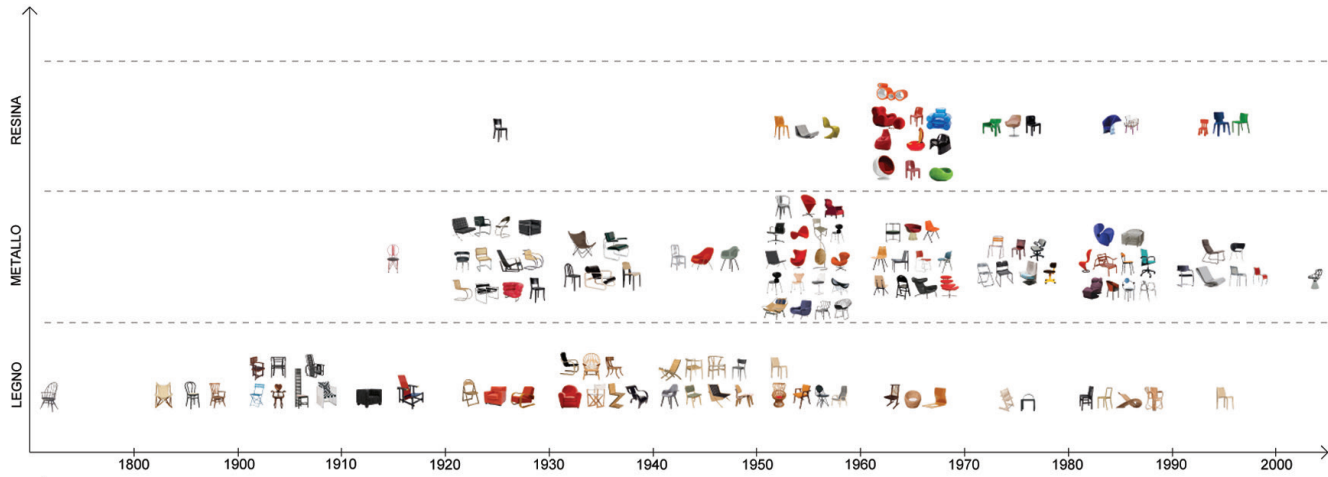

A

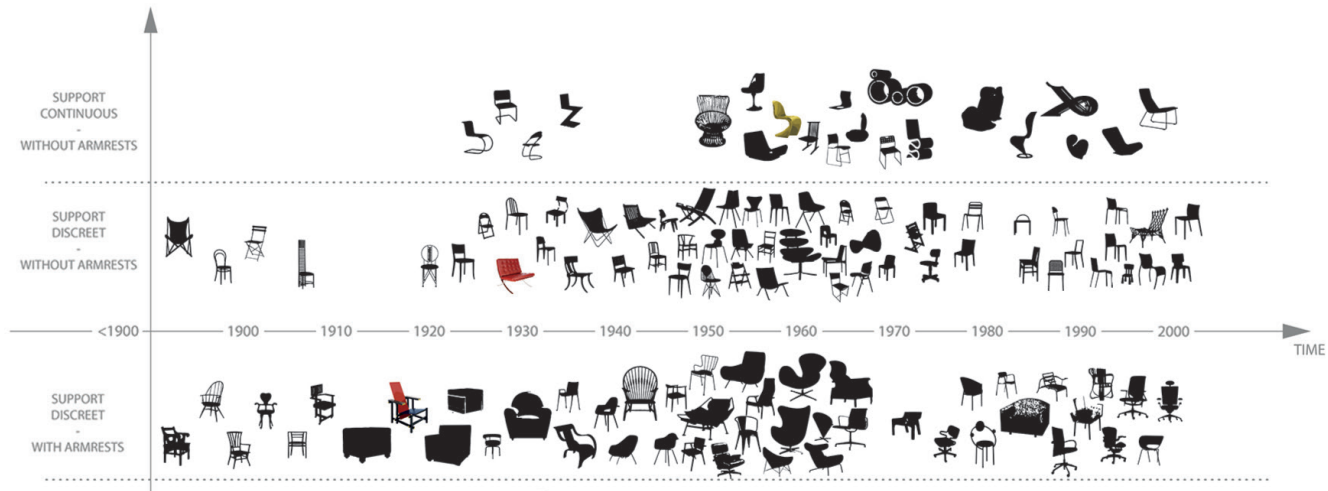

WITHARMRESTS

B
Criteri oggettivi

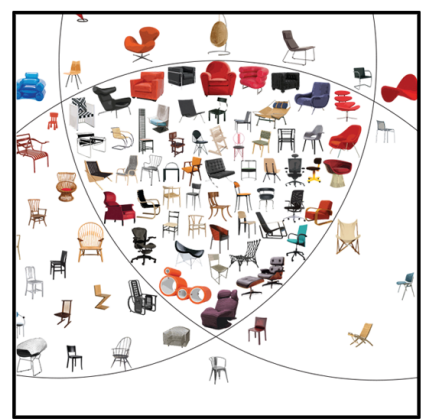

Criteri funzionali
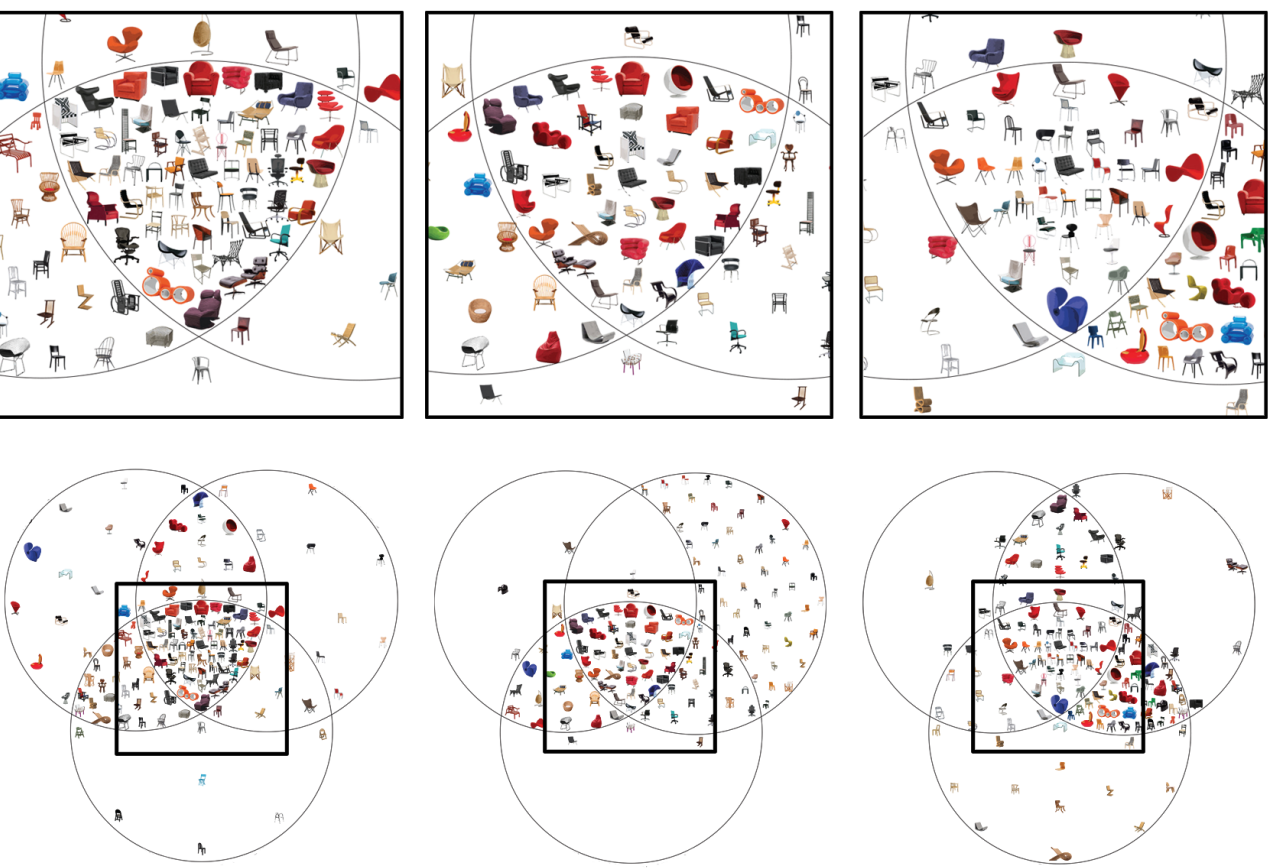
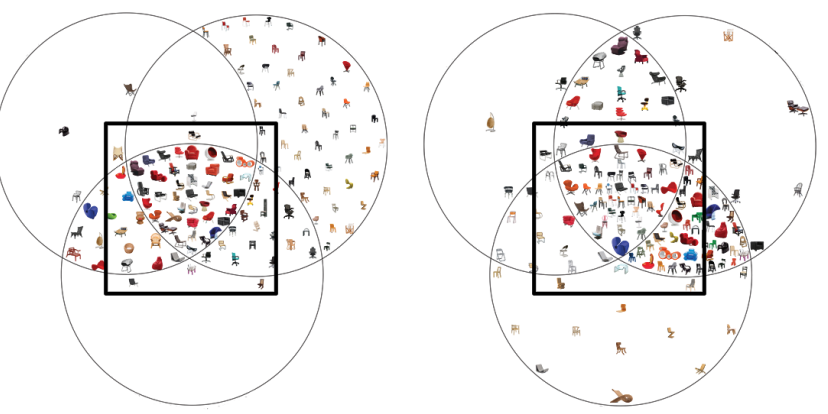
significato non risiede più nell'oggetto stesso in quanto immediatamente percepibile, ma nella correlazione dei segni fra di loro" [Prestinenza Puglisi 1998, p. 39].

Nell'anno 1969 viene esposta a Philadelphia Étant donnés, installazione di M. Duchamp. Quest'opera, un evento rappresentato nel mondo reale, ci interessa per il meccanismo di interattività con il quale l'opera stessa coinvolge il suo osservatore. Étant donnés, infatti, rivela il proprio senso profondo solo a chi, avvicinandosi alla porta in legno, è in grado di individuare i due spioncini attraverso i quali, poi, è possibile osservare la parte nascosta e più significativa dell'installazione stessa.

T. Kawamata è un artista che propone tra il 1983 e il 2005 la serie di maquette da titolo Tree hut plan. Lo scopo della loro realizzazione risiede nella sperimentazione dei meccanismi che successivamente verranno applicati alle sue opere. Sono strumenti di riflessione che intervengono nel processo creativo permettendo all'artista di prefigurare alcune importanti caratteristiche delle sue installazioni.

Tutti gli esempi citati (fig. 2), parte di un più ampio bagaglio di esemplificazioni, consentono una efficace riflessione sull'interpretazione artistica di tematiche, per noi fondamentali, quali il passaggio dalla condizione di realtà a quella di virtualità, o ancora sulla possibilità di favorire un coinvolgimento attivo nell'interpretazione dell'opera da parte del suo fruitore. Esemplificazioni, queste, che hanno agevolato sia il percorso di formazione degli studenti che il perseguimento degli obiettivi loro assegnati.

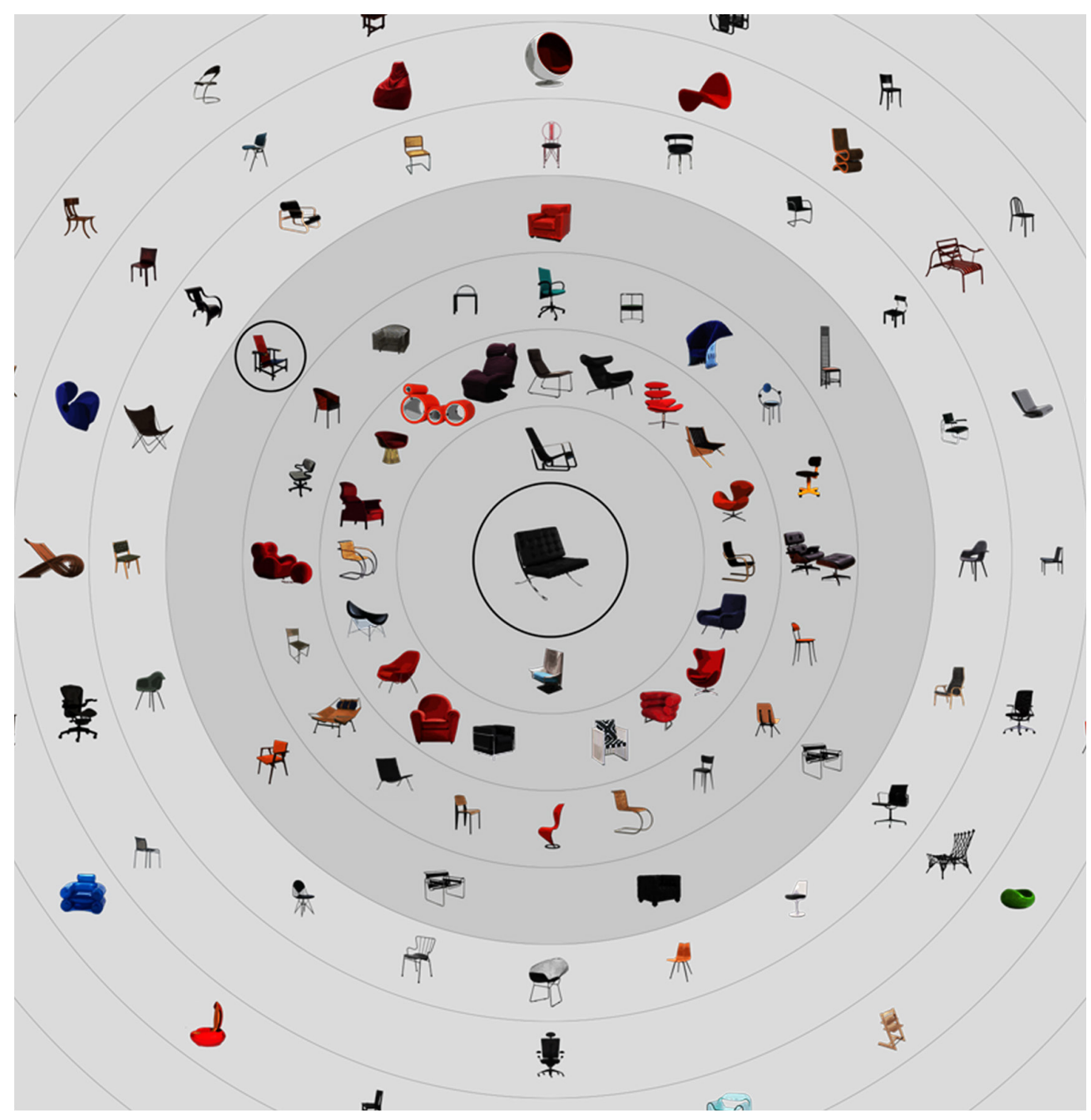




\section{Risultati attesi}

Ispirata al Teorema di Migliari, circoscritta alla rappresentazione codificata dai 12 gradi di iconicità di A. Moles, la nostra ricerca propone il 'Disegno Complesso' come sistema finale e sintetico di rappresentazione dei temi proposti.

Se sul Teorema e sul 'Disegno Complesso' abbiamo già scritto, resta da approfondire il tema dalla scala di iconicità e delle implicazioni generate, per noi, dalla sua applicazione. La scala propone ad un estremo l'oggetto fisico ( $12^{\circ}$ grado), all'altro la descrizione testuale (grado 0), in mezzo le rimanenti rappresentazioni codificate. In sintesi, potremmo dire che il principio applicato alla scala definisce il suo grado di iconicità come l'inverso del livello di astrazione rappresentato. Una interessante conseguenza dell'enunciato, per noi, riguarda la consapevolezza che ad un elevato grado di iconicità corrisponde una maggiore capacità del disegno di descrivere gli aspetti concreti dell'artefatto. All'opposto, la rappresentazione dei suoi aspetti immateriali e concettuali è demandata alle rappresentazioni codificate nei primi posti della scala di iconicità (fig. 3).

Concludendo, quindi, è demandato al 'Disegno Complesso' il compito di integrare l'analisi dell'artefatto alla descrizione delle idee e dei processi sottesi. Ė, ancora, il 'Disegno Complesso' a consentire la lettura simultanea degli elaborati attraverso operazioni di sovrapposizione, contrapposizione e giustapposizione degli elaborati.

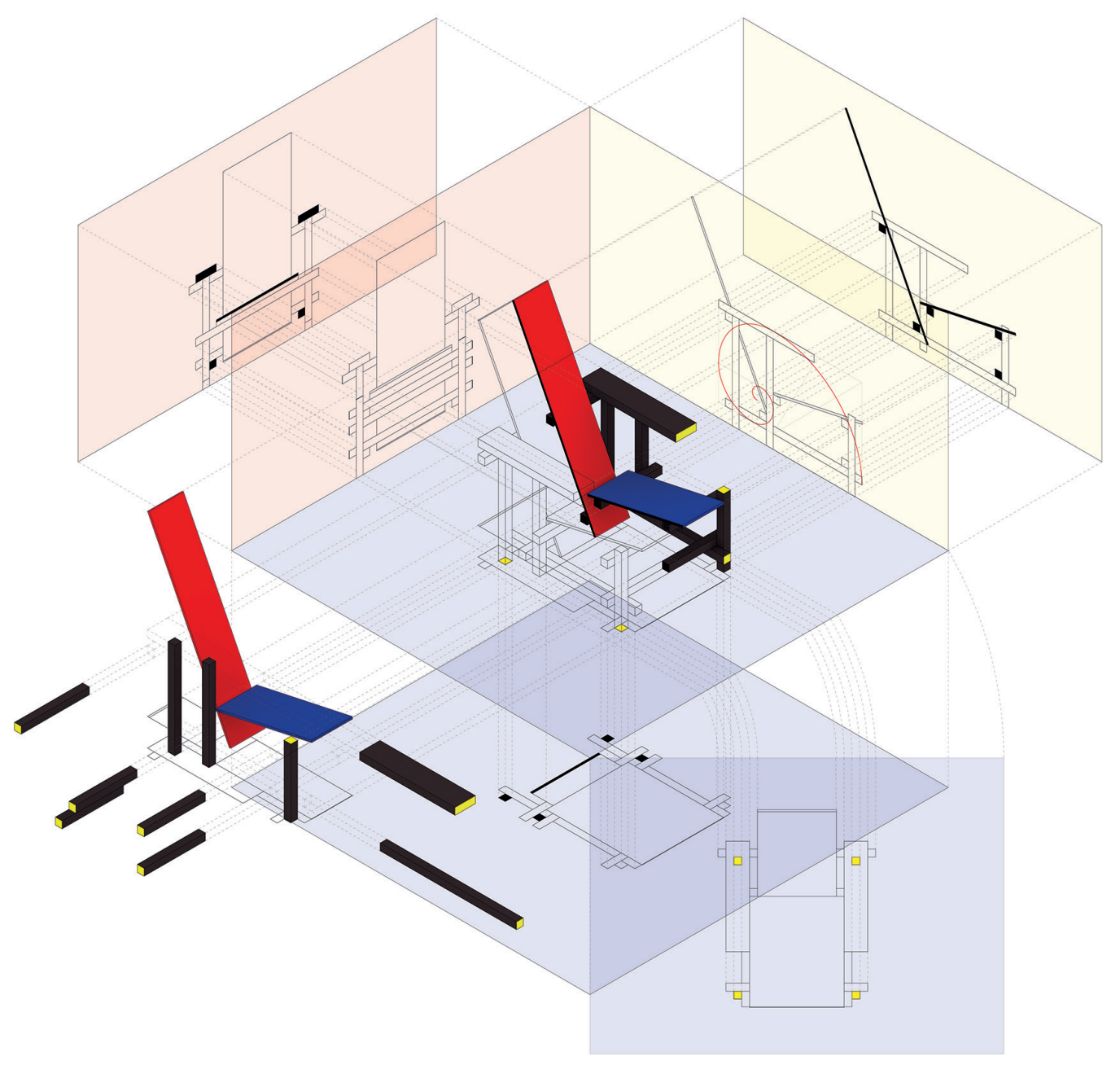




\section{Il tema del corso}

Il tema del corso, ribadiamo, propone di integrare ai canonici disegni dell'artefatto la rappresentazione di quella componente intangibile del progetto, frutto dell'applicazione di un pensiero creativo unico e originale. Nel caso specifico ci occupiamo del Movimento Moderno e di due suoi esponenti: Mies van der Rohe e di GerritThomas Rietveld. La scelta del periodo storico deriva dalla necessità di proporre agli studenti un campo di analisi estremamente connotato, ma sufficientemente circoscritto. Siamo ancora lontani dalle produzioni contemporanee, polverizzate in un caleidoscopio infinito di idee, progetti e prodotti. Gli architetti, quindi, sono stati scelti in quanto validi esponenti di una forma di pensiero pervasivo, integrato e totalizzante, sintetizzato dal Bauhaus nel motto "dal cucchiaio alla città": un sistema di progettazione unitario, frutto di una rigorosa analisi funzionale. I due maestri, quindi, aderiscono al Movimento Moderno e ne accettano le idee generali. Ma come tutti i loro contemporanei personalizzano i concetti di semplificazione della forma, eliminazione delle decorazioni e di abbandono della tradizione naturalistica, in linee di ricerca autonome e originali, oggi conosciute con i nomi di Razionalismo, Funzionalismo, Art Déco, De Stijl ecc. Il corso, in sintesi, propone lo studio di due coppie di manufatti: la sedia Barcelona e il Padiglione tedesco all'Esposizione Universale del 1929 (Mies van der Rohe); la sedia Red\&Blue e la casa Rietveld Schröder (Gerrit Thomas Rietveld). Viene, quindi, chiesto agli studenti di affrontare la lettura del tema indagato in tre livelli di approfondimento. II primo, il più generale, ricerca le relazioni che intercorrono tra il Movimento Moderno e l'intera produzione culturale del XX secolo. II secondo, intermedio, si occupa delle relazioni che intercorrono tra i due architetti, le opere scelte e le rispettive correnti di pensiero. II terzo ed ultimo analizza, seguendo le indicazioni del Bauhaus, quel sistema di progettazione integrato e unitario applicabile sia alla sedia che all'architettura.

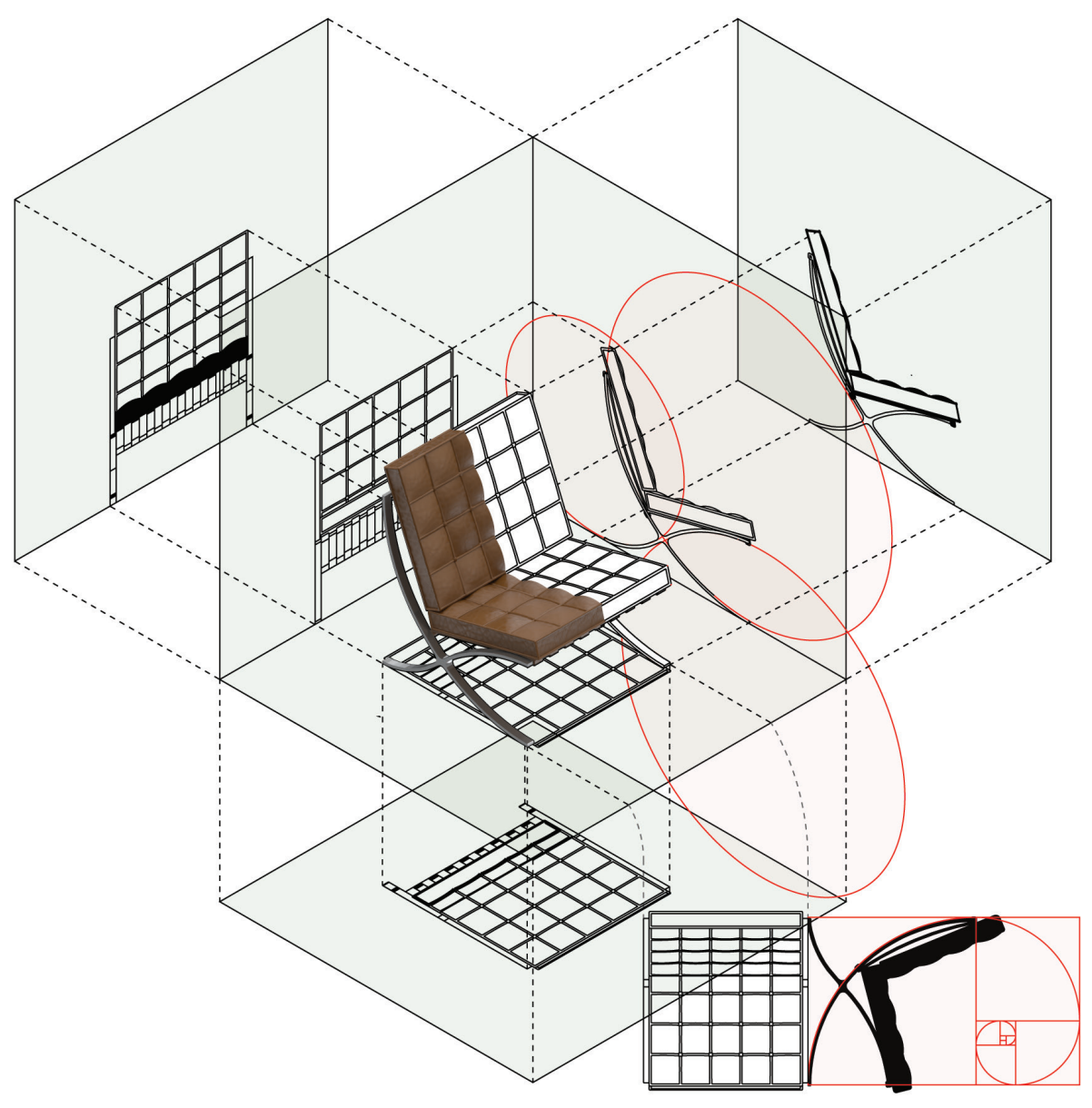


Fig. I0. Stampa 3D della sedia Barcelona.

Fig. I I. Concept di disegno complesso della sedia Barcelona.

Fig. 12. Realizzazione tridimensionale (installazione) del disegno complesso della sedia Barcelona.

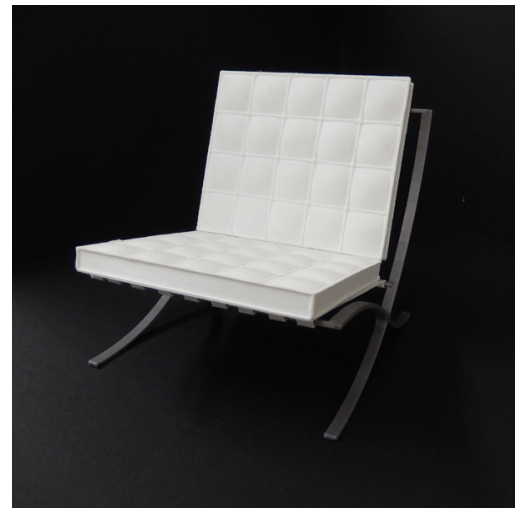

\section{Elaborati realizzati}

La prima parte dello studio viene svolta con l'analisi di I 54 sedie 'd'autore' [2], catalogate in funzione di un certo numero di criteri divisi, a loro volta, in tre categorie di riferimento: oggettiva, percettiva e funzionale (fig. 4).

La stessa natura degli artefatti giustifica il ricorso alla categoria del design per descrivere il fenomeno nei suoi aspetti più generali: ridotte dimensioni, progetti e realizzazioni articolate ed iconiche.

Seguendo la progressione dettata dalla scala di Moles, appartengono a questa prima fase due elaborazioni: un testo scritto e la costruzione di un grafico cartesiano 'in XY' (fig. 5).

II secondo livello valuta invece le relazioni che intercorrono tra gli architetti, le opere e le rispettive correnti di pensiero. Sono state a tale scopo assegnate due sedie, la Barcelona e la Red\&Blue, a cui viene successivamente aggiunta una terza, la Panton, dell'omonimo designer, la prima sedia in plastica stampata al mondo. La necessità di analizzare tre sedie, di cui solo due riconducibili al Movimento Moderno, ha consentito di allargare la comparazione ad un più vasto ambito di progetti consentendone valutazioni e confronti tanto degli aspetti simili che di quelli difformi. Operazione necessaria a rendere plasticamente visibile le trasformazioni epocali imposte dai cambiamenti nella produzione, nelle tecnologie, nel gusto e nella cultura. Appartengono a questo secondo livello due prodotti: una elaborazione grafica basata sulla costruzione di insiemi (fig. 6) e la realizzazione di un grafo finale (fig. 7).

Il terzo ed ultimo livello si occupa della descrizione del sistema di progettazione integrato e unitario applicabile ai due prodotti dello stesso architetto. Ricevono maggiore attenzione in questa fase i disegni bidimensionali e tridimensionali, i render, le fotografie (figg. 8, 9) e gli oggetti prototipati (fig. I0). II corso si conclude con un ultimo atto sostanziale: la costruzione del 'Disegno Complesso' (figg. I I, I2).
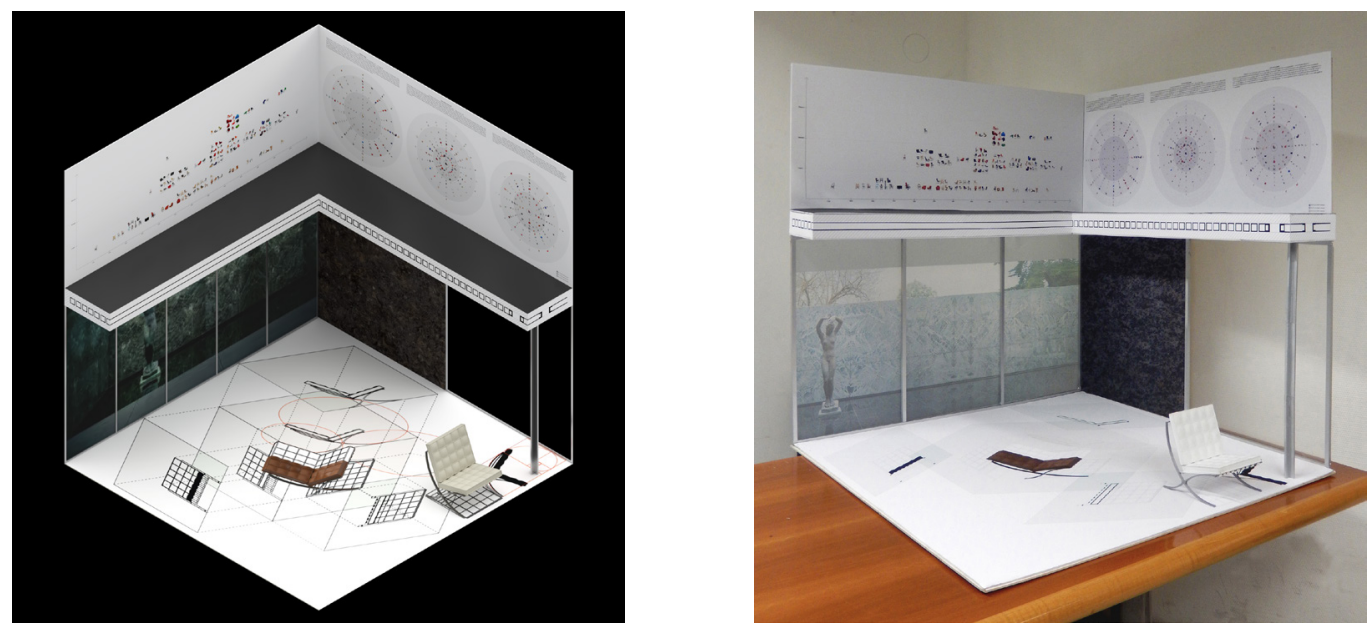


\section{Ringraziamenti}

Si ringraziano gli studenti del corso di Modellazione e Prototipazione A.A. 2019/2020. In particolare gli elaborati fig. 5A-7, 9- I2 sono a cura di B. Basile, S. D'Amico, C. Foti e fig. 5B e 8 a cura di M. Abdelrahman, M. Addario, G. Ibrahim, M. Nawareg e R. Runci.

\section{Note}

[I] "II Teorema esplora le possibili rappresentazioni $(\mathrm{m})$ dell'idea progettuale $(\mathrm{M})$ e ne definisce le relazioni [...] Tutti gli $m$ hanno il medesimo rapporto con $\mathrm{M}$ e dovrebbero essere indicati con un termine generale che li abbracci tutti. Si può usare il termine modelli (scritto con la m minuscola) [...], ma, meglio ancora, è estendere il significato della parola disegno. Ecco dunque il senso dell'enunciato: Disegno come Modello, ovvero Modello, idea, che si invera nelle mutevoli forme del disegno" [Migliari, 2004, pp. 9, 10]

[2] Le I54 sedie 'd'autore' sono estrapolate dai libri II Secolo del Design di C. McDermott, edito da Logos (200 I) e The Design Book (trad. Anna Bissanti) edito da L'ippocampo edizioni (2016 - ed. it.).

\section{Riferimenti bibliografici}

Anceschi Giovanni (1992). L'oggetto della raffigurazione. Milano: Etas.

Arnheim Rudolf (1997). Arte e percezione visiva.Trad. a cura di Gillo Dorfles. Milano: Feltrinelli Editore.

Bradbury Dominic (2019). Modernismo. Arredi, design e grafica 1920-1950. Traduzione a cura di C. Colbertaldo e V. Meloncelli. Milano: Mondadori Electa.

Leoni Fulvio (200 I). L'architettura della simultaneità. Roma: Maltemi editore.

Migliari Riccardo (a cura di). (2004). Disegno come modello. Roma: Edizioni Kappa.

Prestinenza Puglisi Luigi (1998). Hyperarchitettura. Spazi nell'età dell'elettronica. Collegno (To): Universale di Architettura.

Autori

Agostino Urso, Università degli Studi Mediterranea di Reggio Calabria, agostino.urso@unirc.it

Francesco De Lorenzo, Università degli Studi Mediterranea di Reggio Calabria, fdelorenzo@unirc.it

Per citare questo capitolo: Urso Agostino, De Lorenzo Francesco (2020). Due esempi di didattica sulla rappresentazione di relazioni che legano opere, architetti e correnti culturali/Two examples of didactics on representation of the connection among works, architects and cultural currents. In Arena A., Arena M. Brandolino R.G., Colistra D., Ginex G., Mediati D. Nucifora S., Raffa P. (a cura di). Connettere. Un disegno per annodare e tessere. Atti del $42^{\circ}$ Convegno Internazionale dei Docenti delle Discipline della Rappresentazione/Connecting. Drawing for weaving relationships. Proceedings of the 42 th International Conference of Representation Disciplines Teachers. Milano: FrancoAngeli, pp. 867-888. 


\title{
Two Examples of Didactics on Representation of the Connection among Works, Architects and Cultural Currents
}

\author{
Agostino Urso \\ Francesco De Lorenzo
}

\section{Abstract}

Inspired by Riccardo Migliari's Theorem of the Architecture Model, limited to the representation encoded by the 12 Degrees of Iconicity by Abraham Moles, our research proposes the Complex Design as a privileged tool for comparison in the dynamics of the course of study. Using as an example the experience of the Modern Movement, in the interpretation of Mies van der Rohe (the Barcelona chair and the German Pavilion at the Universal Exposition in 1929) and of Gerrit Thomas Rietveld (the Red\&Blue chair and the Schröder house), a pervasive, integrated and totalizing form of thought is told, summarized by the Bauhaus in the slogan "from the spoon to the city": a unitary design system, the result of a strict functional analysis. In conclusion, then, the 'Complex Design' is entrusted with the task of integrating the analysis of the artifact with the description of the ideas and underlying processes, proceeding to deal with the reading of the investigated theme in three levels of depth. The first, the more general one, researches the relationships between the Modern Movement and the entire cultural production of the 20th century. The second one, intermediate, deals with the relationships between the two architects, the chosen works and the respective currents of thought. The third and final one analyzes, following the indications of the Bauhaus, that integrated and unitary design system applicable to both design and architecture.

Keywords

didactics, connections, science of representation, multimedia, architecture.

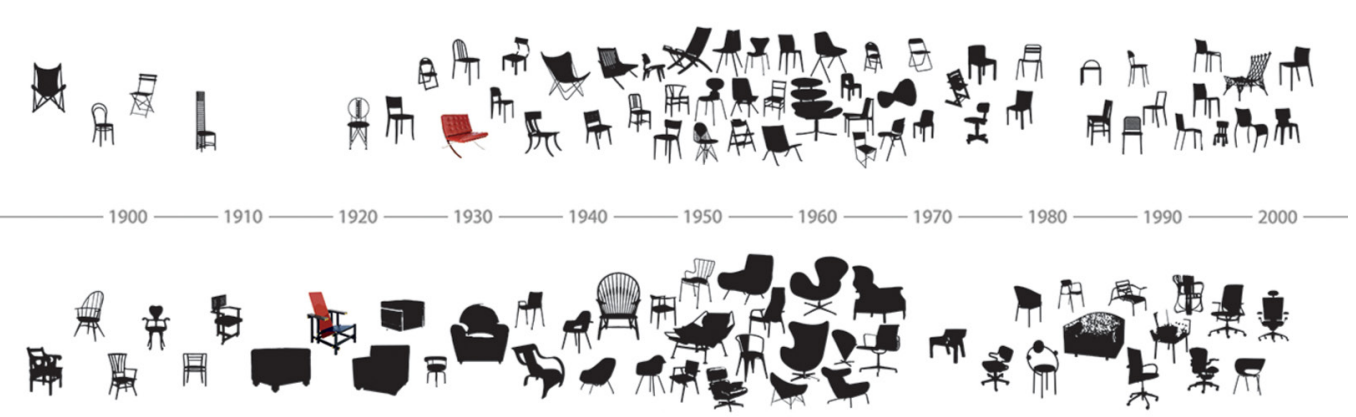




\section{A statement}

It is clear to anyone who has been teaching for a number of years how much the teaching dynamics have changed in the courses of study. You can not deal with the mutation occurred in the interaction between teachers and students without considering the merits of the social changes experienced in recent years. The myth of speed has been replaced by that of simultaneity in which the sharing of information online is now instantaneous and global. By reducing these dynamics to two iconic images, we have on the one hand the teacher, attributable to the root paradigm, on the other the student, plastically defined by the neural network image. The first embodies the traditional attitude of the scholar to deepen each topic, and then summarize and systematize the mutual formal and functional relationships. The learner proceeds in the opposite way: less inclined to reflection, he uses the network to produce fast manipulation of information on the subject investigated, often based on different cultural views. In conclusion, therefore, if the former uses an incremental approach to knowledge, the latter prefers a study method based on the search for meanings and connections generated by networks of information, and then, if necessary, he deepens.

\begin{tabular}{|c|c|c|c|}
\hline \multicolumn{4}{|c|}{ Escala de Iconicidad decreciente de Abraham Moles } \\
\hline Nivel & Definición & Criterio & Ejemplos \\
\hline 12 & $\begin{array}{l}\text { El referente fisico } \\
\text { mismo }\end{array}$ & Iconicidad. & $\begin{array}{l}\text { Objeto en vitrina o en } \\
\text { exposición }\end{array}$ \\
\hline 11 & $\begin{array}{l}\text { Modelo bi- o } \\
\text { tridimensional a } \\
\text { escala }\end{array}$ & Colores y materiales arbitrarios & $\begin{array}{l}\text { Reconstrucción ficticia, } \\
\text { maqueta }\end{array}$ \\
\hline 10 & $\begin{array}{l}\text { Esquema bi-o } \\
\text { tridimensional } \\
\text { reducido o } \\
\text { aumentado } \\
\end{array}$ & $\begin{array}{l}\text { Colores y materiales escogidos según } \\
\text { criterios lógicos }\end{array}$ & $\begin{array}{l}\text { Mapas en } 3 \\
\text { dimensiones,globo terráqueo }\end{array}$ \\
\hline 9 & $\begin{array}{l}\text { Fotografia o } \\
\text { proyección realista } \\
\text { en un plano }\end{array}$ & $\begin{array}{l}\text { Proyección perspectiva rigurosa, medios } \\
\text { tonos y sombras }\end{array}$ & Catálogos ilustrados; posters \\
\hline 8 & $\begin{array}{l}\text { Dibujo, fotografia de } \\
\text { alto contraste }\end{array}$ & $\begin{array}{l}\text { Continuidad del contorno y cierre de la } \\
\text { forma }\end{array}$ & $\begin{array}{l}\text { Afíches, catálogos, } \\
\text { fotografias técnicas }\end{array}$ \\
\hline 7 & Esquema anatómico & $\begin{array}{l}\text { Corte en la carocería o envoltorio; } \\
\text { respeto por la topografia; cuantificación } \\
\text { de elementos y simplificación }\end{array}$ & $\begin{array}{l}\text { Corte anatómico, corte de un } \\
\text { motor, plano de conoxiones } \\
\text { eléctricuas, mapa geográfico }\end{array}$ \\
\hline 6 & $\begin{array}{l}\text { Representaciön } \\
\text { "estallada" }\end{array}$ & $\begin{array}{l}\text { Disposición perspecpectiva artificial de } \\
\text { piezas según sus relaciones de vecindad } \\
\text { topográfica }\end{array}$ & $\begin{array}{l}\text { Objetos técnicos en } \\
\text { manuales de ensamble o } \\
\text { reparación }\end{array}$ \\
\hline 5 & $\begin{array}{l}\text { Esquema de } \\
\text { prinoipio }\end{array}$ & $\begin{array}{l}\text { Substitución de los componentes por } \\
\text { simbolos normalizados; paso de la } \\
\text { topografia a la topologga;geometrización' }\end{array}$ & $\begin{array}{l}\text { Mapa de conexiones de un } \\
\text { receptor de TV, mapa } \\
\text { esquematizado del Metro }\end{array}$ \\
\hline 4 & $\begin{array}{l}\text { Organigrama o } \\
\text { esquema de bloque }\end{array}$ & $\begin{array}{l}\text { Los elementos son cajas negras" } \\
\text { funcionales, conectadas lógicamente, } \\
\text { presentación de funciones lógicas }\end{array}$ & $\begin{array}{l}\text { Organigrama de una } \\
\text { empresa, flujograma de un } \\
\text { programa computacional }\end{array}$ \\
\hline 3 & $\begin{array}{l}\text { Esquema de } \\
\text { formulación }\end{array}$ & $\begin{array}{l}\text { Relación lógica, no topológica, en un } \\
\text { espacio no gcométrico, entre elementos } \\
\text { abstractos. Los lazos son simbólicos y } \\
\text { todos los componentes, visibles. }\end{array}$ & $\begin{array}{l}\text { Fórmulas quimicas } \\
\text { desarrolladas, sociogramas }\end{array}$ \\
\hline 2 & $\begin{array}{l}\text { Esquema en } \\
\text { espacios complejos }\end{array}$ & $\begin{array}{l}\text { Combinación en un mismo espacio de } \\
\text { representación de elementos } \\
\text { escquematicos pertenecientes a sistemas } \\
\text { diforentes }\end{array}$ & $\begin{array}{l}\text { Fuerzas y posiciones } \\
\text { geométricas en una } \\
\text { estructura metálica; esquema } \\
\text { de estática; representación } \\
\text { sono }\end{array}$ \\
\hline 1 & \begin{tabular}{|l|} 
Esquema de vectores \\
en espacios \\
puramente \\
abstractos \\
\end{tabular} & $\begin{array}{l}\text { Representación gráfica en un espacio } \\
\text { métrico abstracto, de relaciones entre } \\
\text { tamaños vectoriales }\end{array}$ & $\begin{array}{l}\text { Gráficos vectoriales en } \\
\text { electrotécnica }\end{array}$ \\
\hline 0 & \begin{tabular}{|l|} 
Descripción en \\
pailabras \\
normalizadas o \\
fórmulas algebráicas
\end{tabular} & $\begin{array}{l}\text { Signos abstractos sin conexión } \\
\text { imaginable con el significado }\end{array}$ & Ecuaciones y textos. \\
\hline
\end{tabular}




\section{Two observations}

First observation. In our personal experience, it often happens that, assigning an analysis and redesign exercise of an architectural masterpiece, providing students with only the bibliographical details, they, in a large part of the cases, consider that they have fulfilled the task assigned with the delivery of the canonical two-dimensional and three-dimensional drawings. Only in a few cases they propose further elaborate graphic designs in relation to the study of symmetries, rhythms and proportions. Finally, almost never, we think about the broader framework of relationships that link the artifact to the thought of its creator or even to the movement of ideas to which the architect is ascribable. The analysis exercise of the assigned architecture, therefore, in a significant number of cases, coincides with its metric representation.

Second observation. Still referring to the assigned task, the substantial difference between the analysis and the redrawing of an architectural masterpiece and an equivalent construction manufactured according to "workmanlike" is almost never understood. At that point, it is necessary to open a reflection on the definition of a work of art and therefore of an architectural artefact of significant cultural interest. It therefore becomes useful to specify how the analysis of a masterpiece, in addition to taking into account the dimensional, structural, material and functional aspects of the building, must consider those cultural processes, bearers of intangible values, whose deep appeal is linked to being at the same time reference, memory and symbol of an entire social system or, in any case, of a political, institutional or cultural part of it.

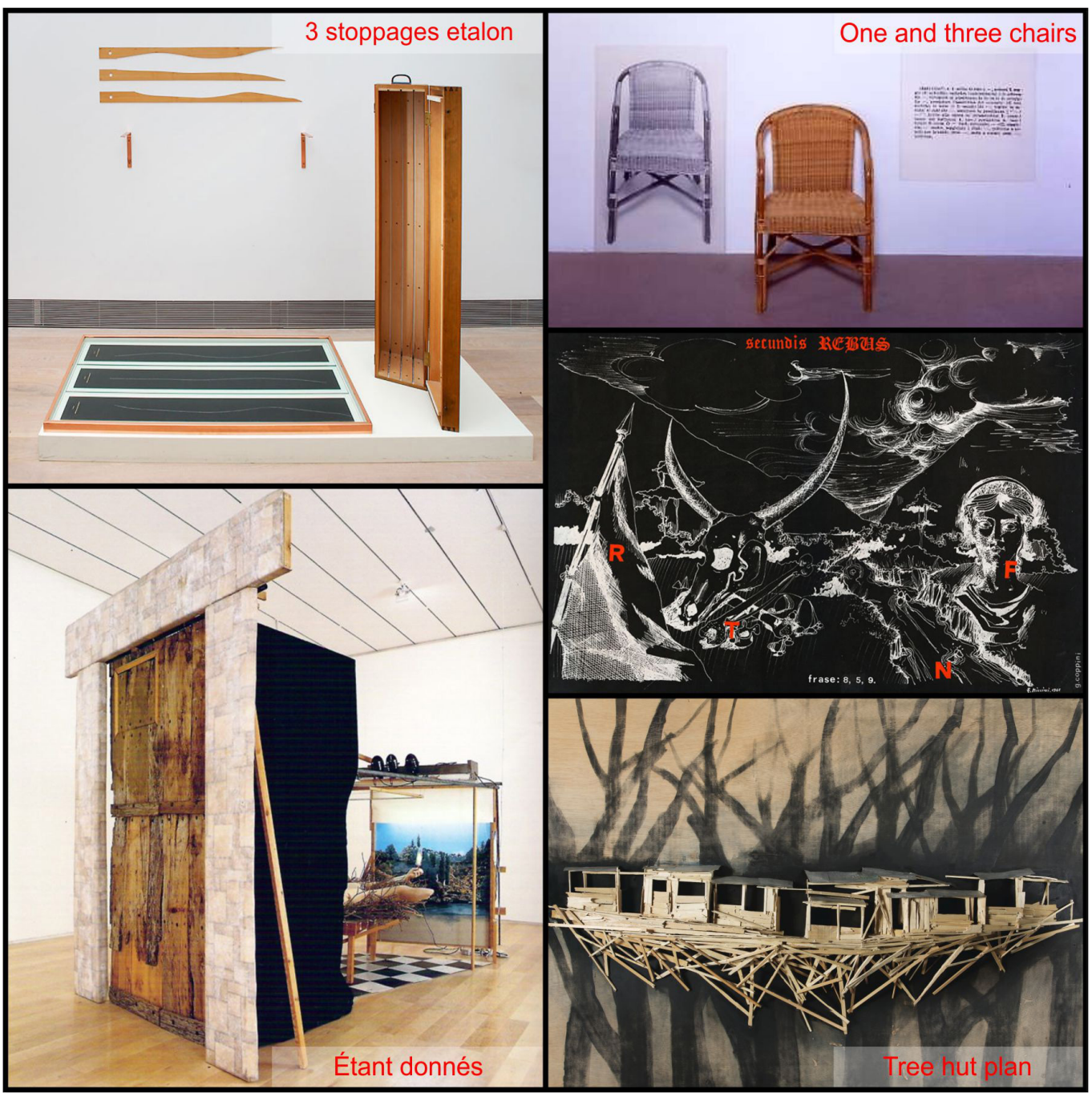




\section{Simultaneous representation of the material and immaterial aspects of an architecture}

On the one hand the two paradigms, the root and the neural network, corresponding to as many methods of acquiring knowledge, on the other a survey practice understood more than as the acquisition of metric data, as redesign of the conveyed intangible relations system. At this point, let's imagine that we can bring the whole of the points of view exercised and the practices used to a synthetic representation that considers both the physical, the perceptual and conceptual aspects: a multimedia approach based on the contemporary reading of the largest number of graphic elaborations produced. Methodology, simplifying a lot, which we can define as the sum of two processes: one theoretical and the other technical. The first promotes the contamination of knowledge through a multidisciplinary comparison. The second identifies, in the Theorem of the Architecture Model [Migliari 2004, pp. 9, I0] [I], a complex system of integrated representations. In essence, the statement of the Theorem could be interpreted as follows: the correspondence between the multimedia synthesis model -which we call 'Complex Design' from this point on- and its artifact will grow as a function of the increase in the number of media used and the respective systematized representations.

On the other hand, the variety of media available and their overlapping poses a serious question about the possible degrees of representation of reality.

For R. Arnheim, representing reality is essentially a problem of visual understanding of form, be it physical or perceptual, to be traced back to the meanings it can express and the contents it intends to communicate.

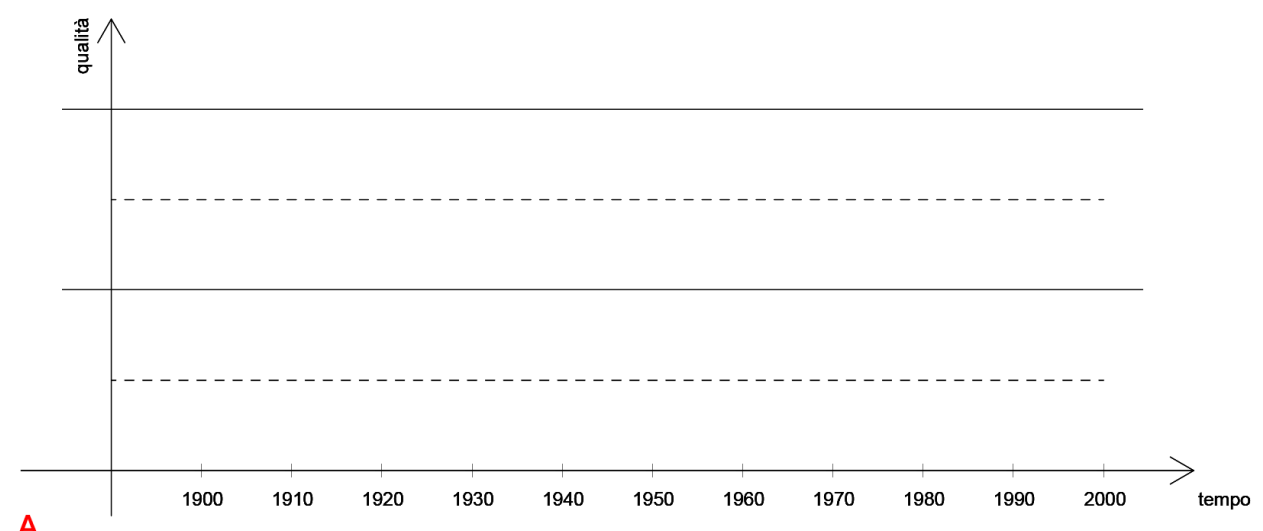

A
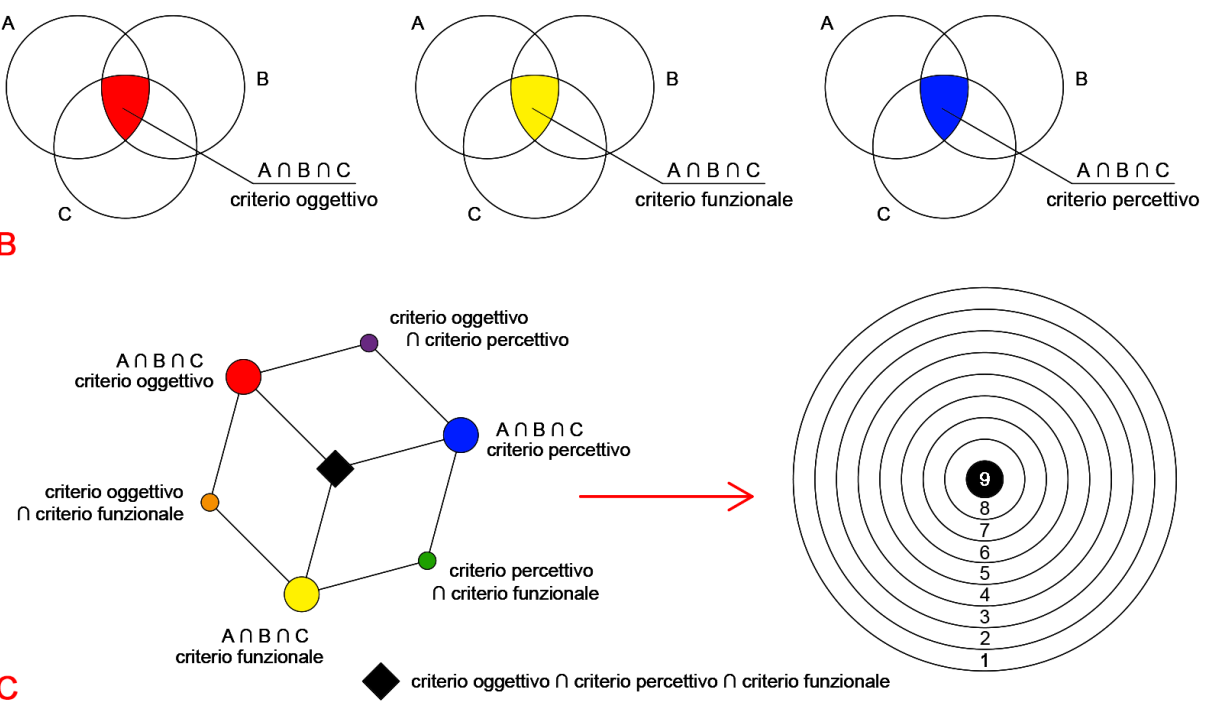
Matt Madden, on the other hand, through the work 99 Ways to Tell a Story: Exercises in Style, it offers an interesting reflection on what relationship of truth exists between the object and its representations. A solution to the theme, already addressed in the 19 th century by Peirce (through the definitions icon, symbol, index), is proposed by Anceschi through the formulation of a unifying theory that includes the scale of iconicity proposed by Abraham Moles. Its 12 degrees include as many variations of representations at whose extremes are positioned the real object and its abstract counterpart, the written word (fig. I). At this point we have an internationally recognized classification, ready to be used to represent the tangible and intangible aspects of any artifact.

\section{Art, representation, multimedia and interaction}

Elaborated in the years 19/3/14 by Marcel Duchamp, the work 3 stoppages étalon is a reflection on the passage from the condition of reality to that of virtuality. This passage produces three interesting consequences: release the subject represented by each of its iconic values; shift attention to its formal relationships; provoke a perceptual alienation sensation in the observer.

The Rebus series -started in 1964 by E. Miccini- at the same time concludes the reflection on multimedia and introduces that on a conceptual approach to interactivity. The Rebus series can be defined as an experimentation that, through continuous transitions between images and words, uses collage as a communication tool for visual poetry. If their messages apparently produce an easy and immediate approval, only an active involvement of its user allows the deciphering of the deepest and most authentic message.

In 1965, J. Kosuth presents the installation "One and three chairs". There are three elements that compose it: a chair, his photograph in a frontal position and a caption with its textual description. Prestinenza Puglisi writes:"By choosing a trivial object and multiplying it by three, Kosuth empties it of every iconic value, with the consequence that its meaning no longer

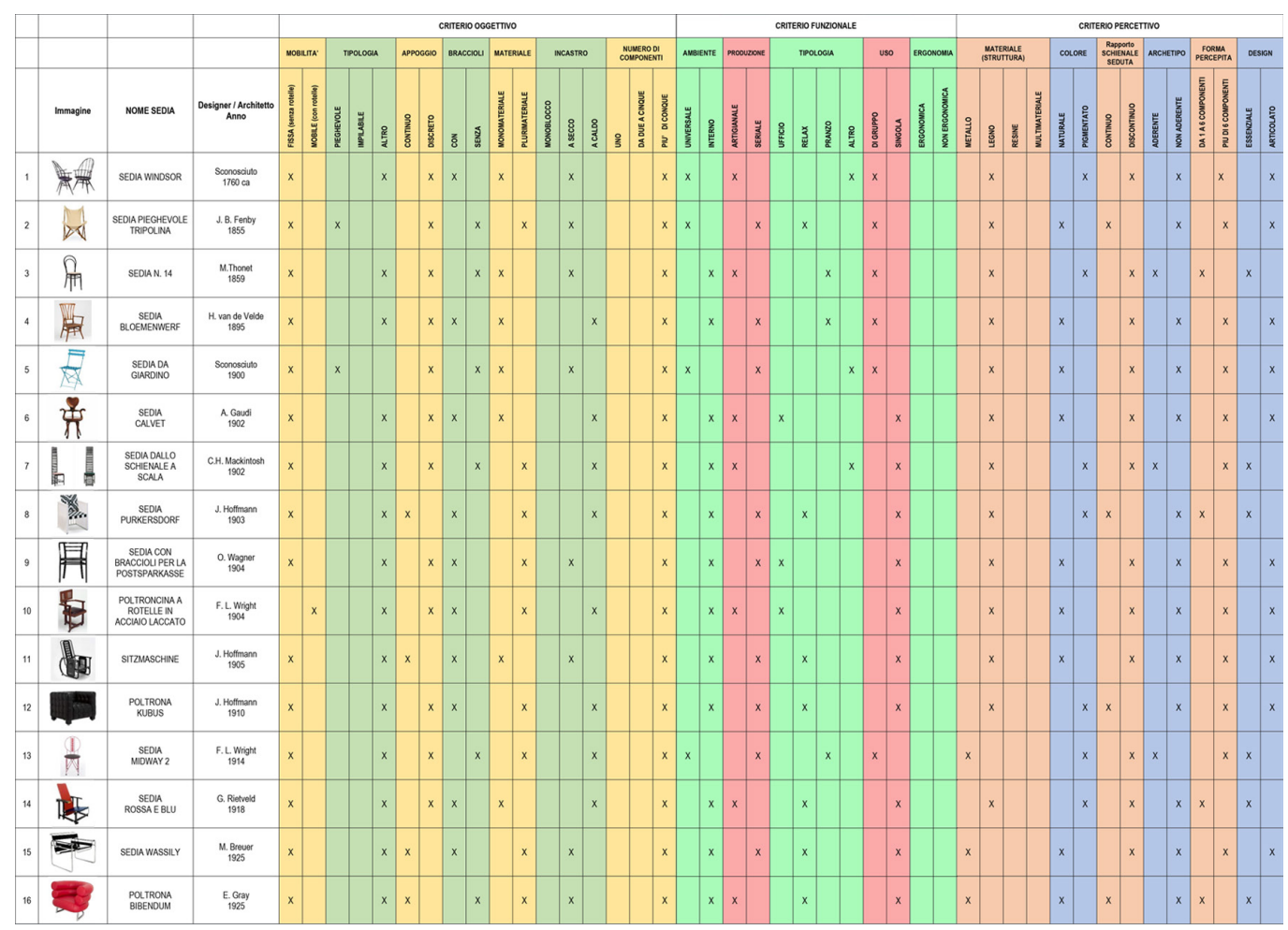


Fig. 5. Cartesian graphic On the abscissa the the ordinate the 'type of material' factor (A) the structure-armrest correlation' factor (B).

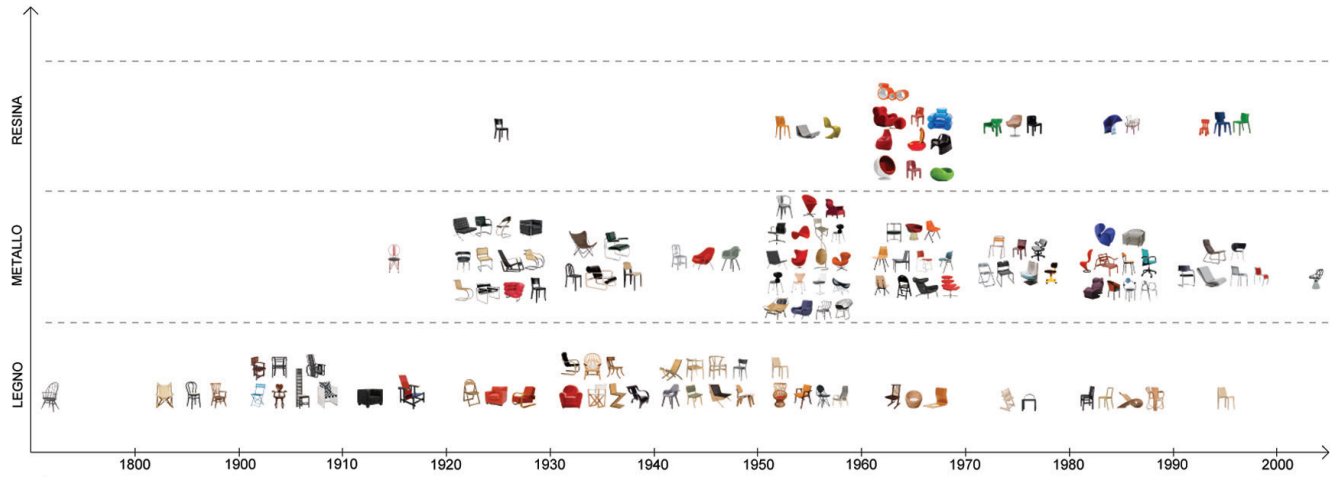

A

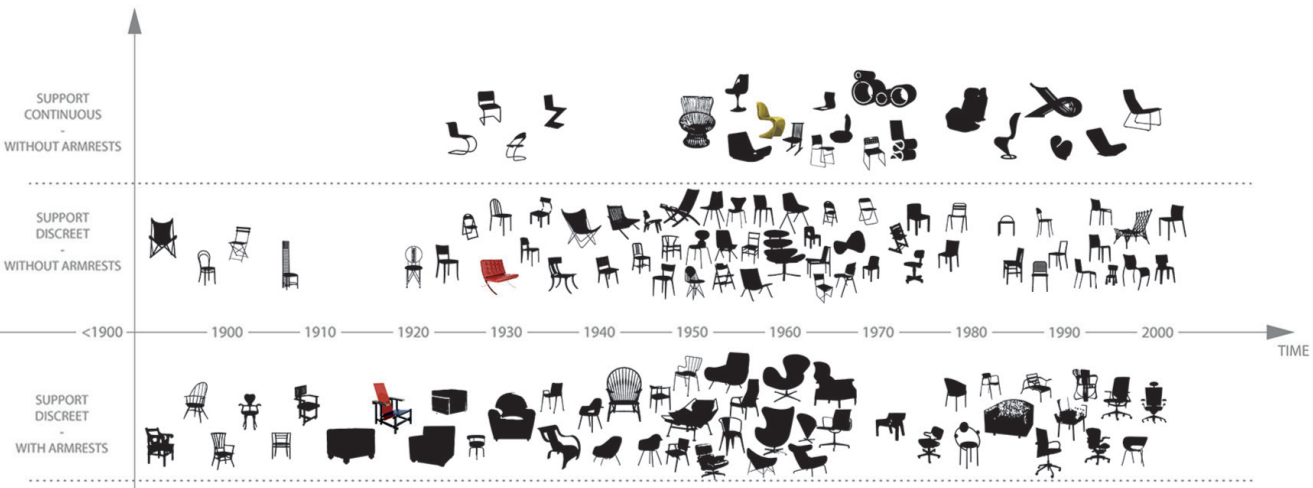

SUPPORT
CONTINUOUS

WITHARMRESTS

B
Criteri oggettivi

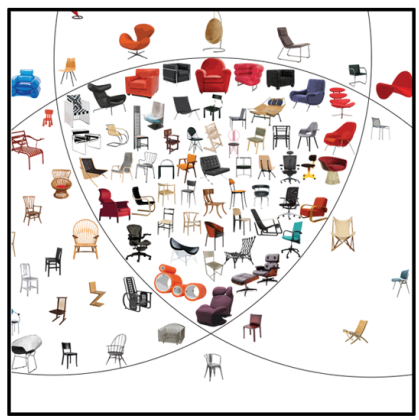

Criteri funzionali
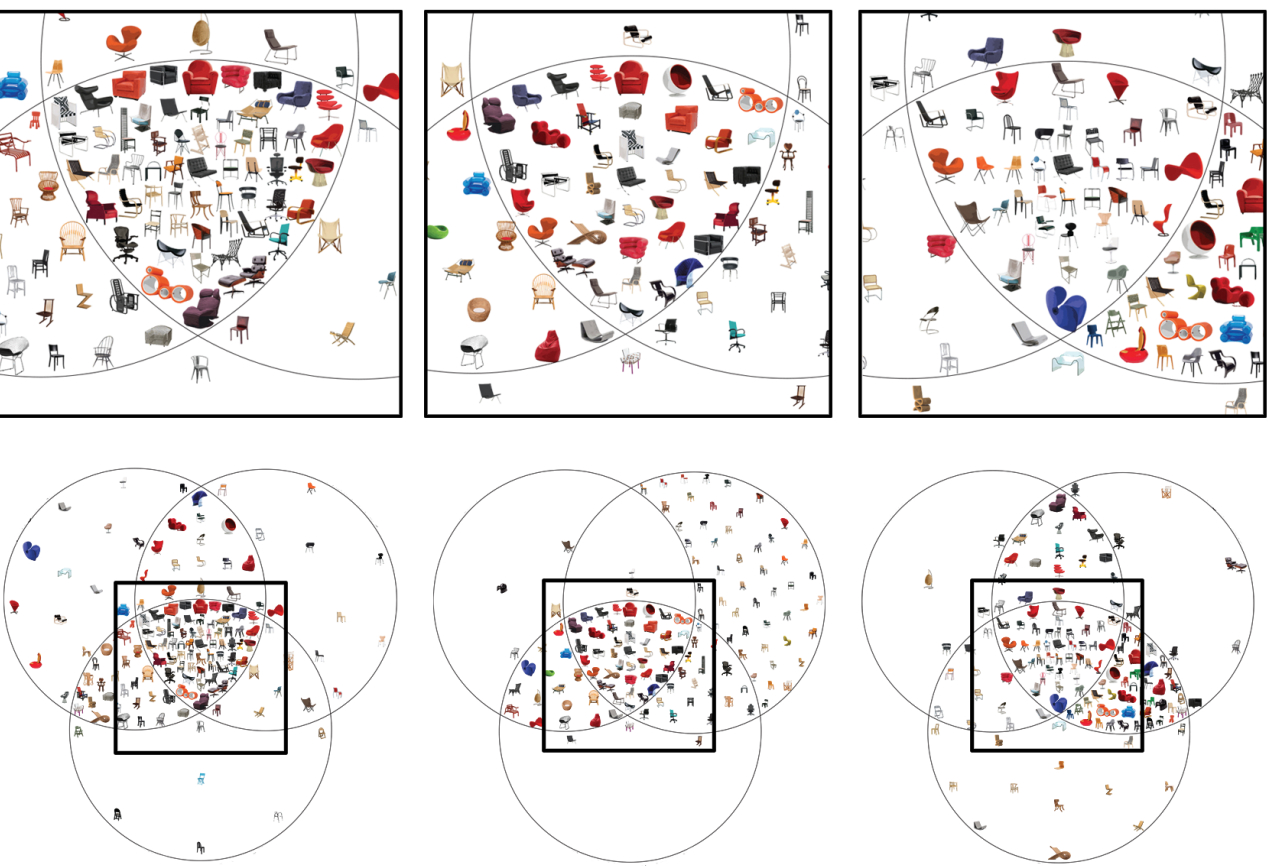
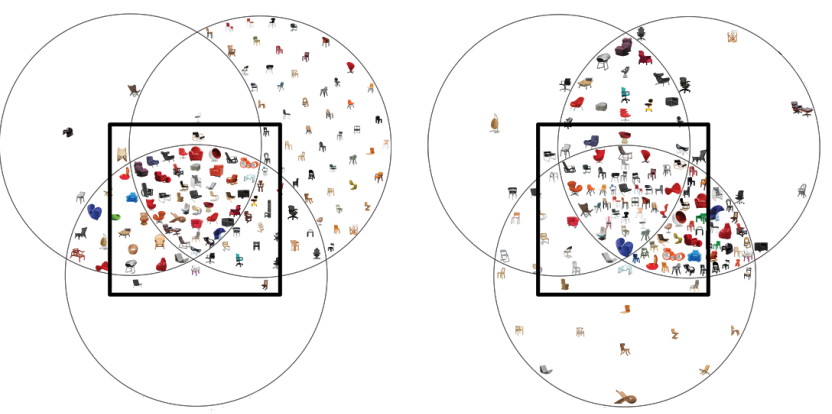
resides in the object itself as it is immediately perceptible, but in the correlation of the signs between them" [Prestinenza Puglisi 1998, p. 39].

In 1969 is exhibited in Philadelphia Étant donnés installation by M. Duchamp. This work, an event represented in the real world, interests us for the interactivity mechanism with which the work itself involves its observer. Étant donnés, in fact, reveals its profound meaning only to those who, approaching the wooden door, are able to identify the two peepholes through which, then, it is possible to observe the hidden and most significant part of the installation itself.

T. Kawamata is an artist who proposes the series of maquettes entitled Tree hut plan between 1983 and 2005. The purpose of their realization lies in the testing of the mechanisms that will subsequently be applied to his works. They are reflection tools that intervene in the creative process, allowing the artist to prefigure some important characteristics of his installations.

All the examples cited (fig. 2), part of a wider range of examples, allow an effective reflection on the artistic interpretation of themes, which are fundamental for us, such as the passage from the condition of reality to that of virtuality, or even on the possibility to encourage an active involvement in the interpretation of the work by its user. Examples, these, which have facilitated both the training path of the students and the pursuit of the objectives assigned to them.

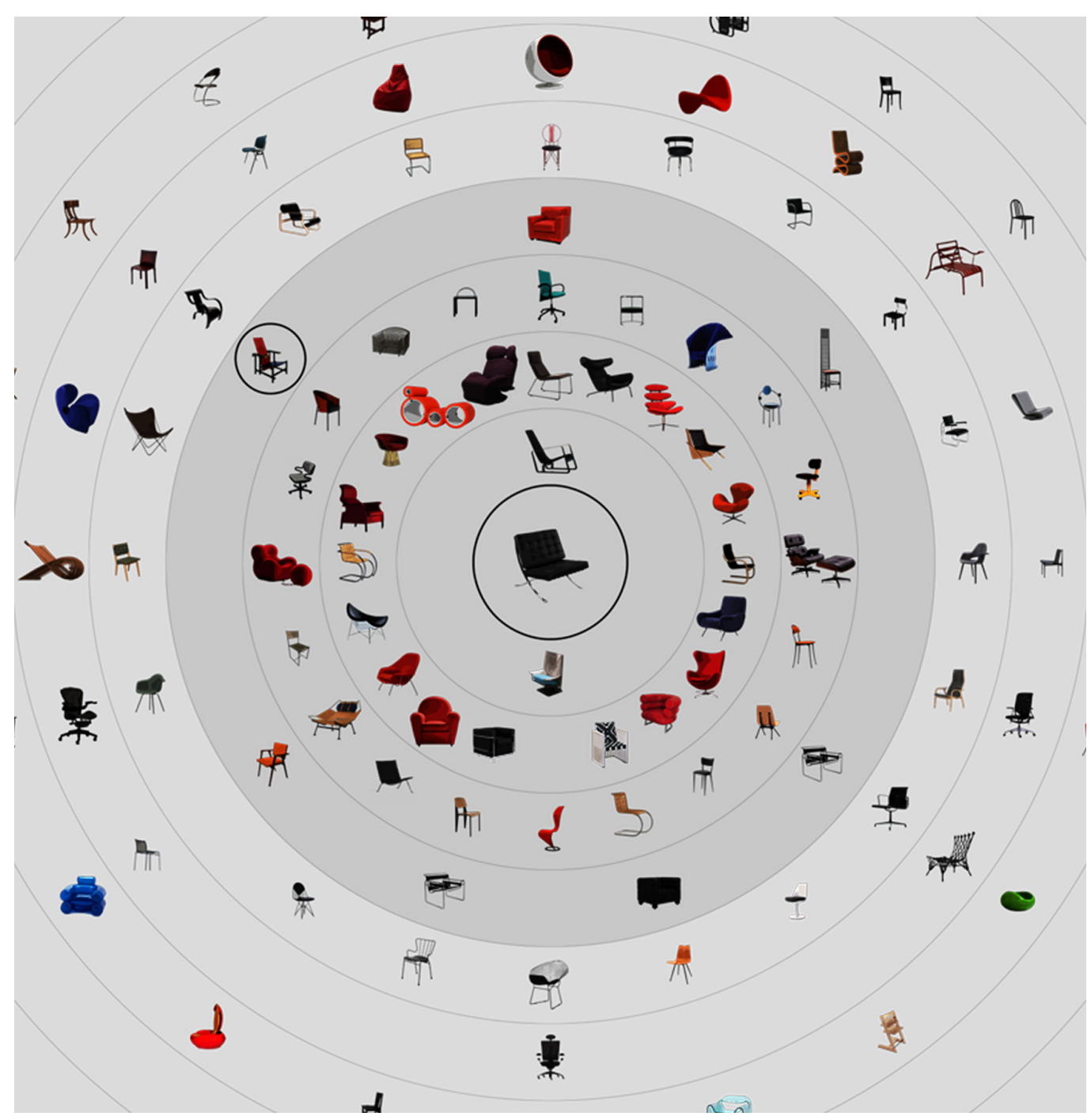




\section{Expected results}

Inspired by Migliari's Theorem, limited to the representation encoded by the 12 degrees of iconicity of A. Moles, our research proposes the 'Complex Design' as a final and synthetic system of representation of the proposed themes.

If we have already written about the Theorem and the 'Complex Design', it remains to deepen the theme of the scale of iconicity and the implications generated, for us, by its application. The scale offers the physical object at one end (I th degree), the textual description at the other (grade 0 ), in the middle the remaining codified representations. In summary we could say that the principle applied to the scale defines its degree of iconicity as the inverse of the level of represented abstraction. An interesting consequence of the statement, for us, concerns the awareness that a high degree of iconicity corresponds to a greater ability of the drawing to describe the concrete aspects of the artifact. On the contrary, the representation of its intangible and conceptual aspects is entrusted to the representations codified in the first places of the iconicity scale (fig. 3).

In conclusion, therefore, the task of integrating the analysis of the artifact with the description of the underlying ideas and processes is entrusted to the 'Complex Design'. It is, again, the 'Complex Design' that allows the simultaneous reading of the works through overlapping, juxtaposition and juxtaposition of the works.

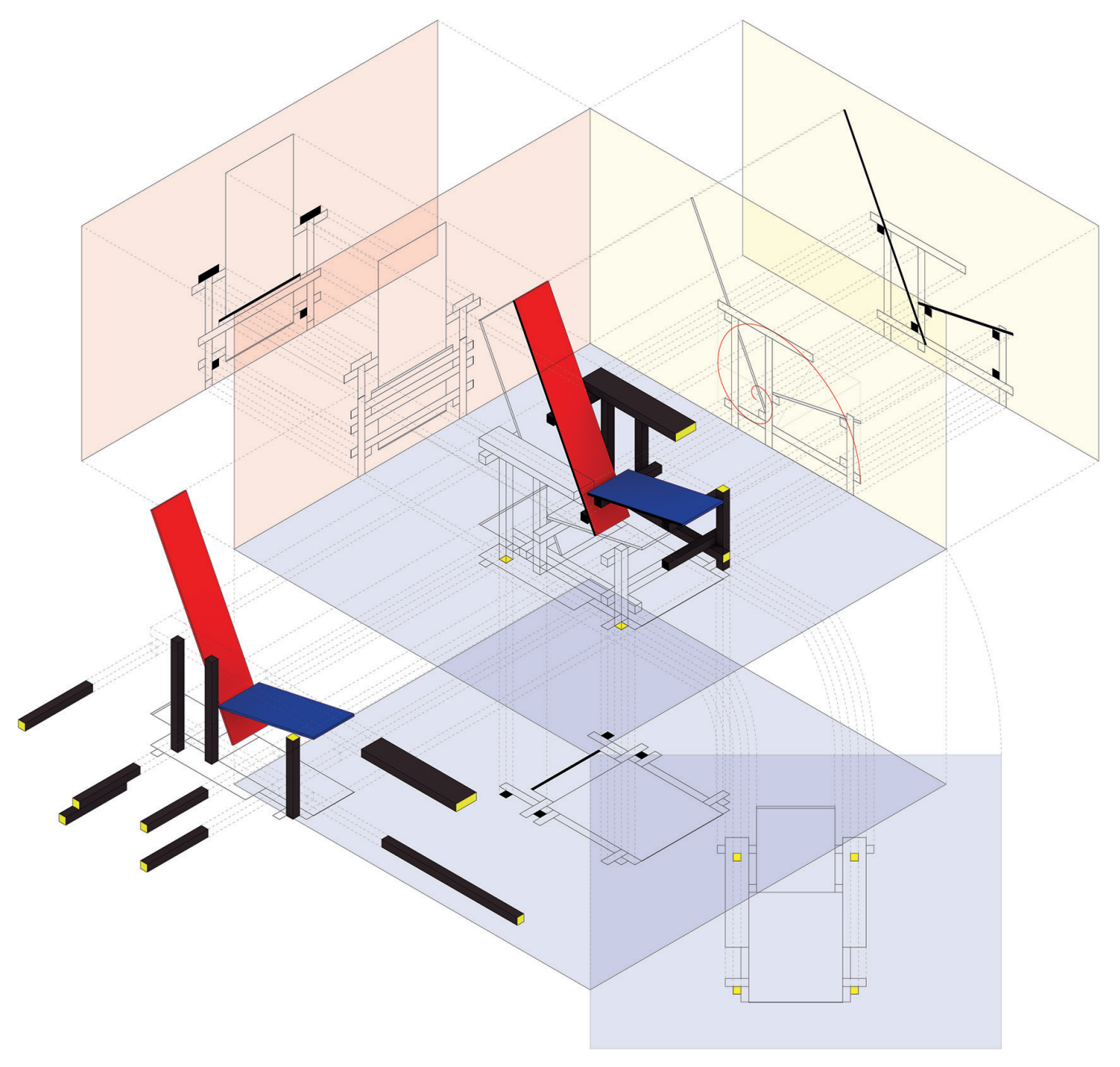




\section{The theme of the course}

The theme of the course, we repeat, proposes to integrate the representation of that intangible component of the project with the canonical drawings of the artifact, the result of the application of a unique and original creative thought. In the specific case, we deal with the Modern Movement and with two of its representatives: Mies van der Rohe and Gerrit Thomas Rietveld. The choice of the historical period derives from the need to offer students an extremely connoted, but sufficiently limited, field of analysis. We are still far from contemporary productions, subdivided in an infinite kaleidoscope of ideas, projects and products. The architects, therefore, have been chosen as valid exponents of a pervasive, integrated and totalizing form of thought, summarized by the Bauhaus in the slogan "from the spoon to the city": a unitary design system, the result of rigorous functional analysis. The two masters therefore adhere to the Modern Movement and accept its general ideas. But like all their contemporaries they personalize the concepts of simplification of form, of elimination of decorations and abandonment of the naturalistic tradition, in autonomous and original lines of research, today known with the names of Rationalism, Functionalism, Art Deco, De Stijl etc. In summary, the course proposes the study of two pairs of artefacts: the Barcelona chair and the German Pavilion at the Universal Exposition in 1929 (Mies van der Rohe); the Red\&Blue chair and the Rietveld Schröder house (Gerrit Thomas Rietveld). It is, therefore, asked the students to face the reading of the theme explored in three levels of study. The first, the more general one, research the relationships between the Modern Movement and the entire cultural production of the twentieth century. The second, intermediate, deals with relations between the two architects, the chosen works and the respective schools of thought. The third and final one analyzes, following the indications of the Bauhaus, that integrated and unitary design system applicable to both the chair and the architecture.

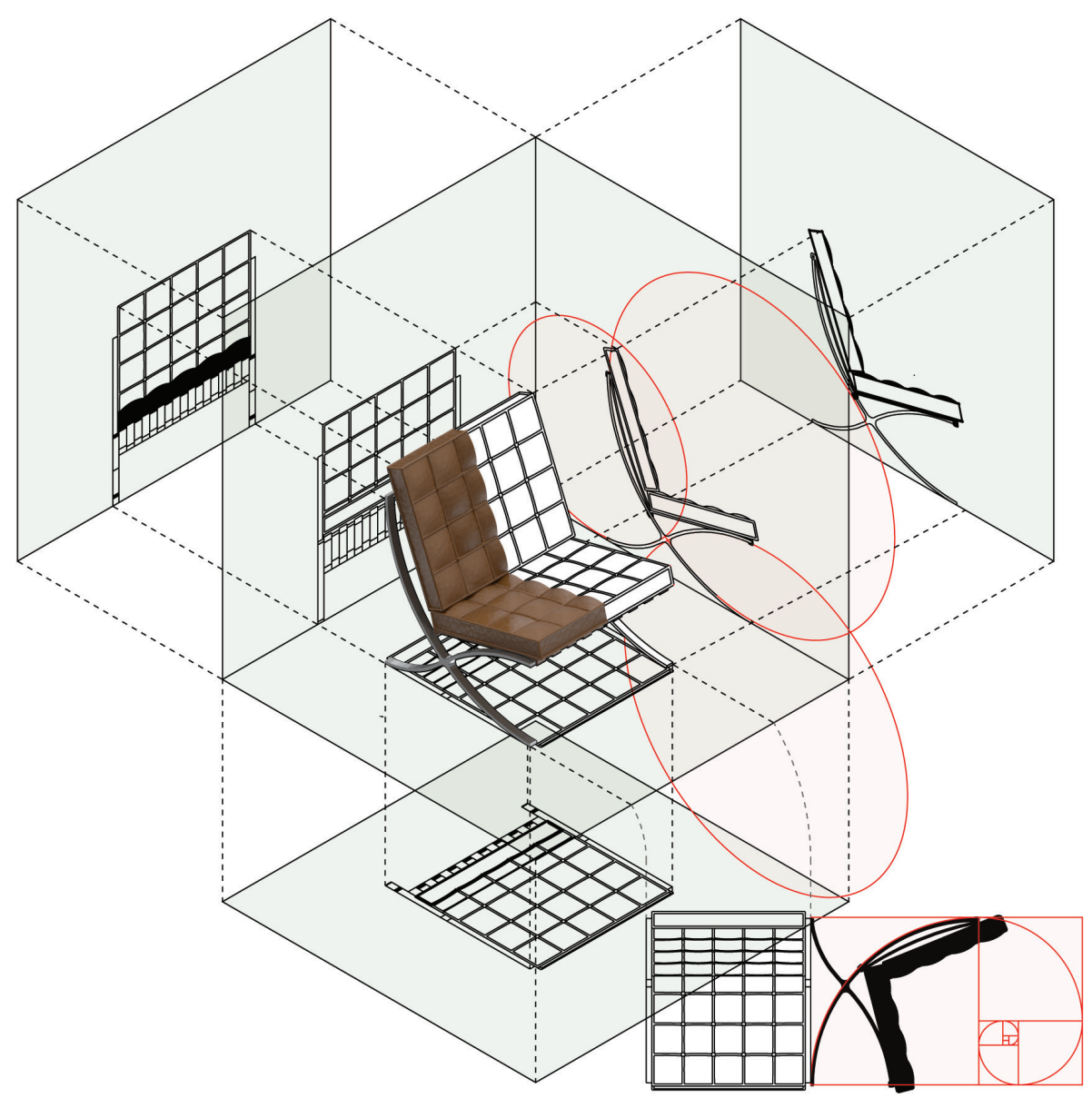



Fig. 10.3D printing of the
Barcelona chair.

Fig. I I. Complex drawing concept of Barcelona chair.

Fig. 12. Three-dimensional realization (installation) realization (Installation)
of the complex drawing of the complex drawing

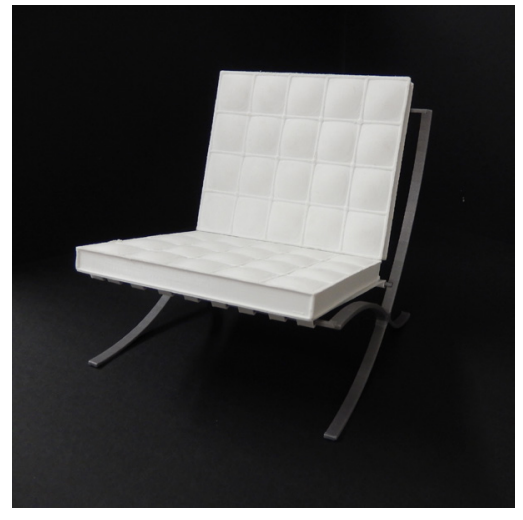

\section{Elaborates that have been made}

The first part of the study is carried out with the analysis of I54 'author's' [2] chairs, cataloged according to a certain number of criteria divided, in turn, into three reference categories: objective, perceptive and functional (fig. 4).

The very nature of the artifacts justifies the use of the category of design to describe the phenomenon in its most general aspects: small dimensions, articulated and iconic projects and creations.

Following the progression dictated by the Moles scale, two elaborations belong to this first phase: a written text and the construction of a Cartesian graph 'in XY' (fig. 5).

The second level, on the other hand, assesses the relationships between architects, their works and their respective currents of thought. Two chairs have been assigned for this purpose, the Barcelona and the Red\&Blue, to which a third is added, the Panton, by the homonymous designer, the first molded plastic chair in the world. The need to analyze three chairs, of which only two attributable to the Modern Movement, has made it possible to increase the comparison to a broader scope of projects, allowing for assessments and comparisons of both similar and different aspects. This operation is necessary to make plastically visible epochal transformations imposed by changes in the production, technology, taste and culture. Two products belong to this second level: a graphic elaboration based on the construction of sets (fig. 6) and the realization of a final graph (fig. 7).

The third and last level deals with the description of the integrated and unitary design system applicable to the two products of the same architect. Two-dimensional and three-dimensional drawings, renders, photographs (figs. 8, 9) and prototyped objects (fig. I0) receive greater attention in this phase. The course concludes with a final substantial act: the construction of the 'Complex Design' (figs. II, 12).
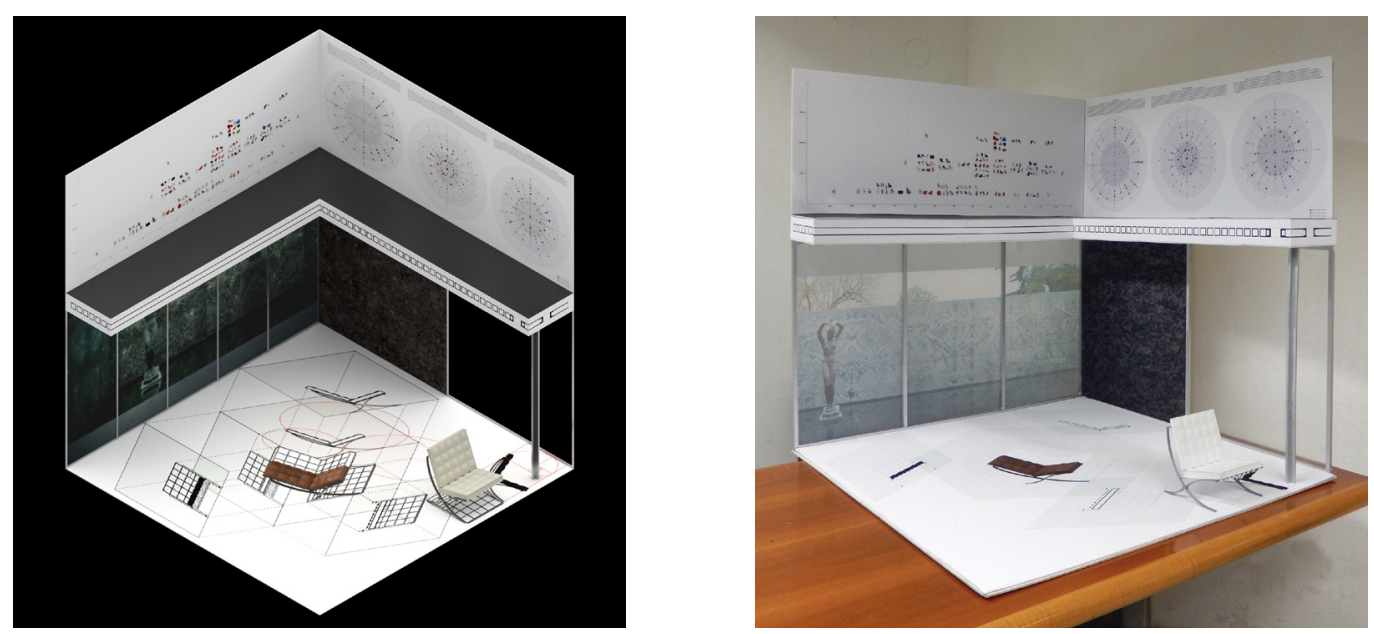


\section{Acknowledgement}

Thanks to the students attending the Modeling and Prototyping course A.A. 2019/2020. In particular, the drawings figs. 5A-7 9-12 are edited by B. Basile, S. D'Amico, C. Foti and fig. 5B and 8 by M. Abdelrahman, M. Addario, G. Ibrahim, M. Nawareg and R. Runci.

\section{Notes}

[I] "The Theorem explores the possible representations $(m)$ of the project idea $(M)$ and defines the relationships. [...] All $m$ have the same relationship with $M$ and should be indicated with a general term that embraces them all. You can use the term models (written with a lowercase $m$ ) è... ], but better yet, it is to extend the meaning of the word drawing. Here is the meaning of the statement: Drawing as a Model, that is, a Model, an idea, which comes true in the changing forms of drawing"'[Migliari, 2004, pp. 9-10].

[2] The I54 'author's' chairs are extrapolated from the books II Secolo del Design by C. McDermott's published by Logos (200 I) and The Design Book (trad. Anna Bissanti) published by L'ippocampo edizioni (2016 - Italian vers.).

\section{References}

Anceschi Giovanni (1992). L'oggetto della raffigurazione. Milano: Etas.

Arnheim Rudolf (1997). Arte e percezione visiva.Trad. a cura di Gillo Dorfles. Milano: Feltrinelli Editore.

Bradbury Dominic (2019). Modernismo. Arredi, design e grafica 1920-1950. Traduzione a cura di C. Colbertaldo e V. Meloncelli. Milano: Mondadori Electa.

Leoni Fulvio (200I). L'architettura della simultaneità. Roma: Maltemi editore.

Migliari Riccardo (a cura di). (2004). Disegno come modello. Roma: Edizioni Kappa.

Prestinenza Puglisi Luigi ( 1998). Hyperarchitettura. Spazi nell'età dell'elettronica. Collegno (To): Universale di Architettura.

\section{Authors}

Agostino Urso, Mediterranea University of Reggio Calabria, agostino.urso@unirc.it

Francesco De Lorenzo, Mediterranea University of Reggio Calabria, fdelorenzo@unirc.it

To cite this chapter: Urso Agostino, De Lorenzo Francesco (2020). Due esempi di didattica sulla rappresentazione di relazioni che legano opere, architetti e correnti culturali/Two examples of didactics on representation of the connection among works, architects and cultural currents. In Arena A., Arena M., Brandolino R.G., Colistra D., Ginex G., Mediati D., Nucifora S., Raffa P. (a cura di). Connettere. Un disegno per annodare e tessere. Atti del $42^{\circ}$ Convegno Internazionale dei Docenti delle Discipline della Rappresentazione/Connecting. Drawing for weaving relationships. Proceedings of the 42 th International Conference of Representation Disciplines Teachers. Milano: FrancoAngeli, Pp. 867-888. 